\title{
LIMITING DEPENDENCE STRUCTURES FOR TAIL EVENTS, WITH APPLICATIONS TO CREDIT DERIVATIVES
}

\author{
ARTHUR CHARPENTIER, ${ }^{*}$ ENSAE/CREST \\ ALESSANDRO JURI,** UBS AG, Zürich
}

\begin{abstract}
Dependence structures for bivariate extremal events are analyzed using particular types of copula. Weak convergence results for copulas along the lines of the Pickands-Balkemade Haan theorem provide limiting dependence structures for bivariate tail events. A characterization of these limiting copulas is also provided by means of invariance properties. The results obtained are applied to the credit risk area, where, for intensitybased default models, stress scenario dependence structures for widely traded products such as credit default swap baskets or first-to-default contract types are proposed.
\end{abstract}

Keywords: Copula; credit risk; dependent defaults; dependent risks; extreme value theory; regular variation; tail dependence

2000 Mathematics Subject Classification: Primary 62E20

Secondary 62H20; 62P05

\section{Introduction}

The reasons for studying and modeling dependencies in finance and insurance are of different types. One motivation is that independence assumptions, which are typical of many stochastic models, are often due more to convenience than to the nature of the problem at hand. Furthermore, there are situations where the neglect of dependence effects may incur a (dramatic) risk underestimation (see, e.g. Bäuerle and Müller (1998) and Daul et al. (2003)). Besides this, widely used scalar dependence or risk measures such as linear correlation, taildependence coefficients, and value at risk generally do not provide a satisfactory description of the underlying dependence structure and have severe limitations when used for measuring (portfolio) risk outside the Gaussian world (see, e.g. Embrechts et al. (2002) and Juri and Wüthrich (2004) for counterexamples).

Taking care of dependencies therefore becomes important in extending standard models to provide more efficient risk management. However, relaxing the independence assumption yields much less tractable models. It is therefore not surprising that only recently, i.e. within the last ten years, has the mathematical literature on the risk management of dependent risks undergone significant development. The main message of much of this research is the following (see, e.g. Dhaene and Goovaerts (1996), Dhaene and Denuit (1999), Frees and Valdez (1998), Joe (1997), Schönbucher and Schubert (2001), and Juri and Wüthrich (2002), (2004), among others). It is (intuitively) clear that the probabilistic mechanism governing the interactions

Received 3 November 2004; revision received 30 January 2006.

* Postal address: Laboratoire de Finance et Assurance, ENSAE/CREST, Timbre J120, 3 avenue Pierre Larousse, FR-92245 Malakoff Cedex, France. Email address: arthur.charpentier@ensae.fr

** Postal address: Credit Risk Control, UBS AG, PO Box, CH-8098 Zürich, Switzerland.

Email address: alessandro.juri@ubs.com 
between random variables is completely described by their joint distribution. On the other hand, in most applied situations, the joint distribution may be unknown or difficult to estimate, so that only the marginals are known (estimated or fixed a priori). To tackle this problem, a flexible and powerful approach consists in trying to model the joint distribution by means of copulas. The latter, which are often called dependence structures, can be viewed as marginal free versions of joint distribution functions capturing scale-invariant dependence properties of the random variables.

The drawback to the copula approach is that it is usually difficult to choose or find the appropriate copula for the problem at hand. Often, the only possibility is to start with some guess, such as a parametric family of copulas, and then try to fit the parameters (as in, e.g. Daul et al. (2003)). As a consequence, the models obtained may suffer from a certain degree of arbitrariness. As was shown by Juri and Wüthrich (2002), (2004), a partial remedy to this weakness of the copula approach is provided by dependence models for (bivariate) conditional joint extremes, from which limiting results along the lines of the Pickands-Balkema-de Haan theorem are obtained. Such 'copula-convergence theorems' reflect a distributional approach to the modeling of dependencies in the tails and provide natural descriptions of multivariate extremal events. Moreover, they differ from classical bivariate extreme value results, since the limits obtained are not bivariate extreme value distributions. A further advantage of these kinds of results is that they can also allow us to better face the problem of the lack of data, which is typical for rare events. In fact, there are situations in which the knowledge of the limiting dependence structure reduces the issue of modeling tail events to the estimation of only one parameter (Juri and Wüthrich (2002)).

\subsection{Outline of the paper}

The paper is structured as follows. In Section 2.1 we briefly recall the copula concept and all of its properties that we will need in the rest of the paper. The idea of dependence structures for tail events is then formalized in Section 2.2, where the concept of a lower tail-dependence copula (LTDC) is introduced; this provides a natural description of conditional bivariate joint extremes. Sections 3 and 4 contain the main results, which extend some of the work of Juri and Wüthrich (2002), (2004). In particular, under suitable regularity conditions, Theorem 3.1 identifies possible LTDC limits, i.e. limit laws for bivariate joint extremes. Motivated by classical results such as the central limit theorem and the Fisher-Tippett theorem, we show in Section 4 that LTDC limits are characterized by invariance properties (see Theorems 4.1 and 4.2 and Corollary 4.1). In Section 5 we show how the results of the preceding sections can be applied to the credit risk area, where, for intensity-based default models, we obtain dependence structures characterizing the behavior under stress scenarios of widely traded credit derivatives such as credit default swap baskets or first-to-default contract types. The proofs of our results, where pertinent, are collected in Section 6.

\section{Dependence structures for tail events}

\subsection{Preliminaries}

As mentioned above, one of the main concepts used to describe scale-invariant dependence properties of multivariate distributions is that of the copula. In this work, we focus on bivariate continuous random vectors only; most of the following preliminary material can be found in Nelsen (1999) or Joe (1997).

Definition 2.1. A two-dimensional copula is a two-dimensional distribution function restricted to $[0,1]^{2}$ with standard uniform marginals. 
Copulas can be equivalently defined as functions $C:[0,1]^{2} \rightarrow[0,1]$ satisfying, for $0 \leq$ $x \leq 1$ and $\left(x_{1}, y_{1}\right),\left(x_{2}, y_{2}\right) \in[0,1]^{2}$ with $x_{1} \leq x_{2}$ and $y_{1} \leq y_{2}$, the conditions

$$
\begin{aligned}
& C(x, 1)=C(1, x)=x, \quad C(x, 0)=C(0, x)=0, \\
& C\left(x_{2}, y_{2}\right)-C\left(x_{2}, y_{1}\right)-C\left(x_{1}, y_{2}\right)+C\left(x_{1}, y_{1}\right) \geq 0 .
\end{aligned}
$$

In fact, it is easily seen that (2.1) translates into the uniformity of the marginals and that (2.2), which is known as the 2-increasing property, can be interpreted as $\mathrm{P}\left[x_{1} \leq X \leq x_{2}, y_{1} \leq\right.$ $Y \leq y_{2}$ ], for a pair $(X, Y)$ having distribution function $C$. Note that (2.2) neither implies nor is implied by the fact that $C$ is increasing in each argument. However, (2.1) together with (2.2) imply that $C$ increases in each variable, and that $C$ is Lipschitz continuous with Lipschitz constant 1 .

One of the most important and useful results about copulas is Sklar's theorem, stated below in its bivariate form. A proof of Theorem 2.1 can be found, e.g. in Nelsen (1999, p. 18) or in Sklar (1959).

Theorem 2.1. (Sklar's theorem.) (i) Let $C$ be a copula and let $F_{1}$ and $F_{2}$ be univariate distribution functions. Then, for $\left(t_{1}, t_{2}\right) \in \mathbb{R}^{2}$,

$$
F\left(t_{1}, t_{2}\right):=C\left(F_{1}\left(t_{1}\right), F_{2}\left(t_{2}\right)\right)
$$

defines a distribution function with marginals $F_{1}$ and $F_{2}$.

(ii) Conversely, for a two-dimensional distribution function $F$ with marginals $F_{1}$ and $F_{2}$, there is a copula $C$ satisfying (2.3). This copula is not necessarily unique, but it is if $F_{1}$ and $F_{2}$ are continuous, in which case, for any $(x, y) \in[0,1]^{2}$,

$$
C(x, y)=F\left(F_{1}^{-1}(x), F_{2}^{-1}(y)\right),
$$

where $F_{1}^{-1}$ and $F_{2}^{-1}$ denote the generalized left-continuous inverses of $F_{1}$ and $F_{2}$, respectively.

Sklar's theorem provides the motivation to refer to copulas as dependence structures that capture scale-invariant dependence properties. In fact, we see from (2.3) that $C$ couples the marginals $F_{1}$ and $F_{2}$ to the joint distribution function $F$, thus separating dependence and marginal behaviors. Further, it is easy to check that for random variables $X_{1}$ and $X_{2}$ with joint distribution function $F$, copula $C$ (in the sense that $C$ is a copula satisfying (2.3)), and strictly increasing functions $f_{1}, f_{2}: \mathbb{R} \rightarrow \mathbb{R}$, the variables $f_{1}\left(X_{1}\right)$ and $f_{2}\left(X_{2}\right)$ also have copula $C$.

An example of a copula is the following. Throughout the rest of the paper we will encounter others.

Example 2.1. The Marshall and Olkin copula with parameters $\alpha, \beta \in[0,1]$ is defined, for $x, y \in[0,1]$, by

$$
C_{\alpha, \beta}(x, y):=\left(x^{1-\alpha} y\right) \wedge\left(x y^{1-\beta}\right),
$$

where, for real numbers $s$ and $t$, the expression $s \wedge t$ denotes their minimum.

\subsection{Tail-dependence copulas}

A natural way to construct dependence structures (copulas) for bivariate (lower) tail events is first to consider two-dimensional, continuous conditional distribution functions where the condition is that both variables fall below small thresholds. The second step is to obtain the corresponding copula using the second part of Sklar's theorem (i.e. (2.4)). 
Remark 2.1. In the sequel, we will assume that the considered copula $C$ is such that the functions $x \mapsto C(x, y)$ and $y \mapsto C(x, y)$ are strictly increasing for all $x, y \in(0,1]$. We denote by $\mathcal{C}$ the set of such copulas.

Let $(U, V)$ be a random vector with distribution function $C \in \mathcal{C}$. For any $(u, v) \in(0,1]^{2}$, the conditional distribution of $(U, V)$ given that $U \leq u$ and $V \leq v$, denoted by $F(C, u, v)$, is given, for $0 \leq x \leq u$ and $0 \leq y \leq v$, by

$$
F(C, u, v)(x, y)=\mathrm{P}[U \leq x, V \leq y \mid U \leq u, V \leq v]=\frac{C(x, y)}{C(u, v)}
$$

The marginal distribution functions of this $F(C, u, v)$ are respectively given, for $0 \leq x \leq u$ and $0 \leq y \leq v$, by

$$
F_{U}(C, u, v)(x)=\frac{C(x, v)}{C(u, v)} \quad \text { and } \quad F_{V}(C, u, v)(y)=\frac{C(u, y)}{C(u, v)} .
$$

Since these two functions are continuous, the unique copula corresponding to $F(C, u, v)$ is obtained from (2.4) and equals

$$
\begin{array}{r}
F(C, u, v)\left(F_{U}(C, u, v)^{-1}(x), F_{V}(C, u, v)^{-1}(y)\right) \\
=\frac{C\left(F_{U}(C, u, v)^{-1}(x), F_{V}(C, u, v)^{-1}(y)\right)}{C(u, v)} .
\end{array}
$$

Definition 2.2. For $C \in \mathcal{C}$, we call the copula defined by (2.7) the lower tail-dependence copula $(L T D C)$ relative to $C$, and denote it by $\Phi(C, u, v)$.

Note that the assumption $C \in \mathcal{C}$ implies that $\left\{(u, v) \in[0,1]^{2}: C(u, v)>0\right\}=(0,1]^{2}$, i.e. it ensures that the LTDC $\Phi(C, u, v)$ is well defined for all $u, v \in(0,1]$. Furthermore, $\lim _{u, v \rightarrow 0} \Phi(C, u, v)$ naturally describes the dependence structure underlying conditional bivariate random samples in the lower tails.

Furthermore, starting with uniform marginals, i.e. with a copula $C$, is not a restriction, since the dependence structure that would be obtained with different marginals is of the same type as $\Phi(C, u, v)$. In fact, let $X_{1}$ and $X_{2}$ have joint distribution function $G$, strictly increasing, continuous marginals $G_{1}$ and $G_{2}$, and copula $C$. Analogously to the above, for appropriate $z_{1}, z_{2} \in \mathbb{R}$ (i.e. such that the following expressions are well defined), consider the conditional distribution function

$$
G^{z_{1}, z_{2}}\left(x_{1}, x_{2}\right):=\mathrm{P}\left[X_{1} \leq x_{1}, X_{2} \leq x_{2} \mid X_{1} \leq z_{1}, X_{2} \leq z_{2}\right]
$$

Furthermore, let $G_{1}^{z_{1}, z_{2}}\left(x_{1}\right):=G^{z_{1}, z_{2}}\left(x_{1}, z_{2}\right)$ and $G_{2}^{z_{1}, z_{2}}\left(x_{2}\right):=G^{z_{1}, z_{2}}\left(z_{1}, x_{2}\right)$. From Sklar's theorem, the copula relative to $G^{z_{1}, z_{2}}$ is given by

$$
\Phi\left(G, z_{1}, z_{2}\right)\left(u_{1}, u_{2}\right):=G^{z_{1}, z_{2}}\left(\left(G_{1}^{z_{1}, z_{2}}\right)^{-1}\left(u_{1}\right),\left(G_{2}^{z_{1}, z_{2}}\right)^{-1}\left(u_{2}\right)\right) .
$$

Proposition 2.1. In the above setting, we have $\Phi\left(C, G_{1}\left(z_{1}\right), G_{2}\left(z_{2}\right)\right)=\Phi\left(G, z_{1}, z_{2}\right)$. 
Remark 2.2. Sometimes it may be more natural to look at dependencies in the upper tails rather than in the lower tails, e.g. in any situation where we are interested in the joint behavior of random variables conditional on high thresholds. In such a case, in (2.5) we could consider the expression $\mathrm{P}[U>x, V>y \mid U>u, V>v]$ instead of $\mathrm{P}[U \leq x, V \leq y \mid U \leq u$, $V \leq v$ ], yielding, by analogy with (2.7), a dependence structure for upper tail events. Such dependence structures can also be obtained by replacing $C$ in Definition 2.2 by the relative survival copula $\hat{C}(x, y):=x+y-1+C(1-x, 1-y), x, y \in[0,1]^{2}$. Indeed, it is easily seen that for a random vector $(X, Y)$ with distribution function $F$, marginals $F_{1}$ and $F_{2}$, and copula $C$, the copula of $(-X,-Y)$ is precisely $\hat{C}$, and that, for $(x, y) \in \mathbb{R}^{2}$,

$$
\mathrm{P}[X>x, Y>y]=\hat{C}\left(1-F_{1}(x), 1-F_{2}(y)\right) .
$$

\section{A limit theorem}

The main result of this section is Theorem 3.1, in which limits of the type $\lim _{t \rightarrow 0} \Phi(C, r(t)$, $s(t))$ are considered. An explicit form for the limit is provided under the assumption that the functions, $r$ and $s$, defining the direction under which the limit is taken satisfy suitable regularity conditions. Furthermore, an example of a nonsymmetric LTDC limit, i.e. a limit obtained under a direction $(r, s)$ with $r \neq s$, is given in Proposition 3.1, where we show that a dependence model in the lower tails may be given by the Marshall and Olkin copula of Example 2.1. As we will see in Section 5, this copula turns out to be a natural model for some credit derivatives.

For our purposes, the concept of regular variation appears to be the appropriate one. A standard reference to the topic of regular variation is Bingham et al. (1987). Results for the multivariate case can also be found in de Haan et al. (1984).

Definition 3.1. A measurable function $f:(0, \infty) \rightarrow(0, \infty)$ is said to be regularly varying at 0 with index $\rho \in \mathbb{R}$ if, for any $x>0$,

$$
\lim _{t \rightarrow 0} \frac{f(t x)}{f(t)}=x^{\rho} .
$$

We then write $f \in \mathcal{R}_{\rho}^{0}$. For $\rho=0$, the function is said to be slow varying at 0 .

Definition 3.2. A measurable function $f:(0, \infty)^{2} \rightarrow(0, \infty)$ is said to be regularly varying at 0 with auxiliary functions $r, s:(0, \infty) \rightarrow(0, \infty)$ if $\lim _{t \rightarrow 0} r(t)=\lim _{t \rightarrow 0} s(t)=0$ and there is a positive, measurable function $\phi:(0, \infty)^{2} \rightarrow(0, \infty)$ such that

$$
\lim _{t \rightarrow 0} \frac{f(r(t) x, s(t) y)}{f(r(t), s(t))}=\phi(x, y) \text { for all } x, y>0 .
$$

We then write $f \in \mathcal{R}(r, s)$ and call $\phi$ the limiting function under the direction $(r, s)$.

Remark 3.1. Definition 3.2 can be easily modified to include functions, such as copulas, having a domain other than $(0, \infty)^{2}$. This ensures, in particular, that the left-hand side of (3.2), below, is well defined.

Theorem 3.1. Let $C \in \mathcal{C} \cap \mathcal{R}(r, s)$ with limiting function $\phi$, and assume that $r$ and s are strictly increasing, continuous functions such that $r \in \mathcal{R}_{\alpha}^{0}$ and $s \in \mathcal{R}_{\beta}^{0}$ for some $\alpha, \beta>0$. Then, for any $(x, y) \in[0,1]^{2}$,

$$
\lim _{t \rightarrow 0} \Phi(C, r(t), s(t))(x, y)=\phi\left(\phi_{X}^{-1}(x), \phi_{Y}^{-1}(y)\right),
$$


where $\phi_{X}(x):=\phi(x, 1)$ and $\phi_{Y}(y):=\phi(1, y)$. Moreover, there is a constant, $\theta>0$, such that $\phi(x, y)=x^{\theta / \alpha} h\left(y x^{-\beta / \alpha}\right)$ for $x>0$, where

$$
h(t):= \begin{cases}\phi_{Y}(t) & \text { for } t \in[0,1], \\ t^{\theta / \beta} \phi_{X}\left(t^{-\alpha / \beta}\right) & \text { for } t \in(1, \infty) .\end{cases}
$$

Remark 3.2. Note that the limiting function, $\phi$, in (3.1) is obtained from a pointwise convergence. Because the domain of a copula is the compact set $[0,1]^{2}$, it follows that the assumption $C \in \mathcal{C} \cap \mathcal{R}(r, s)$ implies that the convergence in (3.2) is uniform, i.e. that

$$
\lim _{t \rightarrow 0}\left\|\Phi(C, r(t), s(t))-\phi\left(\phi_{X}^{-1}(\cdot), \phi_{Y}^{-1}(\cdot)\right)\right\|_{\infty}=0 .
$$

Remark 3.3. Observe that the hypothesis that $r$ and $s$ are continuous functions is a necessary one, since otherwise counterexamples such as copulas with fractal support, as considered by Fredricks et al. (2005), can be constructed. Let $\boldsymbol{T}=\left(t_{i j}\right)$ be a square matrix, with nonnegative entries whose sum equals 1 , determining the following subdivision of the unit square $[0,1]^{2}$ into rectangles. Let $c_{i}, i=0, \ldots, n$, be the sum of the entries of the first $i$ columns of $\boldsymbol{T}$, with $c_{0}:=0$, and let $r_{j}, j=0, \ldots, n$, be the sum of the entries in the first $j$ rows of $\boldsymbol{T}$, with $r_{0}:=0$. Then the vectors $\boldsymbol{r}:=\left(r_{0}, \ldots, r_{n}\right)$ and $\boldsymbol{c}:=\left(c_{0}, \ldots, c_{n}\right)$ define partitions of $[0,1]$, whence $[0,1]^{2}$ is partitioned into the rectangles $R_{i j}:=\left[c_{i-1}, c_{i}\right] \times\left[r_{i-1}, r_{i}\right]$. Furthermore, for a given copula $C$ and a point $(x, y) \in R_{i j}$, consider the new copula, $T(C)$, defined by

$$
\begin{aligned}
T(C)(x, y):= & \sum_{u<i, v<j} t_{u v}+\frac{x-c_{i-1}}{c_{i}-c_{i-1}} \sum_{v<j} t_{i v}+\frac{y-r_{j-1}}{r_{j}-r_{j-1}} \sum_{u<i} t_{u j} \\
& +C\left(\frac{x-c_{i}}{c_{i}-c_{i-1}}, \frac{y-r_{j}}{r_{j}-r_{j-1}}\right) t_{i j},
\end{aligned}
$$

where empty sums are defined to equal 0 . Fredricks et al. (2005) showed that for any copula $C$ and any $\boldsymbol{T} \neq \mathbf{1}$ there is a unique copula, $C_{T}$, that depends only on $\boldsymbol{T}$ and satisfies $T\left(C_{T}\right)=C_{T}$. Moreover, they showed that $C_{T}=\lim _{n \rightarrow \infty} T^{n}(C)$, where $T^{n}(C):=T\left(T^{n-1}(C)\right.$ ) for $n \geq 1$, $T^{1}(C):=T(C)$, and $T^{0}(C):=C$. Now consider the case in which the starting copula $C$ is the independent copula, i.e. $C(x, y)=C^{\perp}(x, y):=x y$, and the transformation matrix is given by

$$
\boldsymbol{T}=\left(\begin{array}{ccc}
0.1 & 0 & 0.1 \\
0 & 0.6 & 0 \\
0.1 & 0 & 0.1
\end{array}\right)
$$

whence $\boldsymbol{c}=\boldsymbol{r}=(0,0.2,0.8,1)$. Then

$$
\Phi\left(C_{T}, t_{k}, t_{k}\right)=C_{T}=\lim _{n \rightarrow \infty} T^{n}(C), \quad \text { where } t_{k}=0.2^{k}, k \geq 1 .
$$

The fact that $\Phi\left(C_{T}, t_{k}, t_{k}\right)=C_{T}$ can be explained with the help of Figure 1, in which the support of $T^{n}(C)$ is plotted for $n=1,2,3,4$ and the shaded regions are those where the measure relative to $T^{n}(C)$ concentrates its mass (indeed, we see from (3.4) that the support of $T^{n}(C)$ is given by the rectangles corresponding to the nonzero elements of $\boldsymbol{T}$ ). Observe that since $C$ is the independent copula, the measure relative to $T^{n}(C)$ spreads its mass uniformly on the shaded squares. Taking, for example, the upper-right panel of Figure 1, we see that restricting ourselves to $\left[0, t_{1}\right]^{2}=[0,0.2]^{2}$ yields exactly the same picture as in the upper-left 

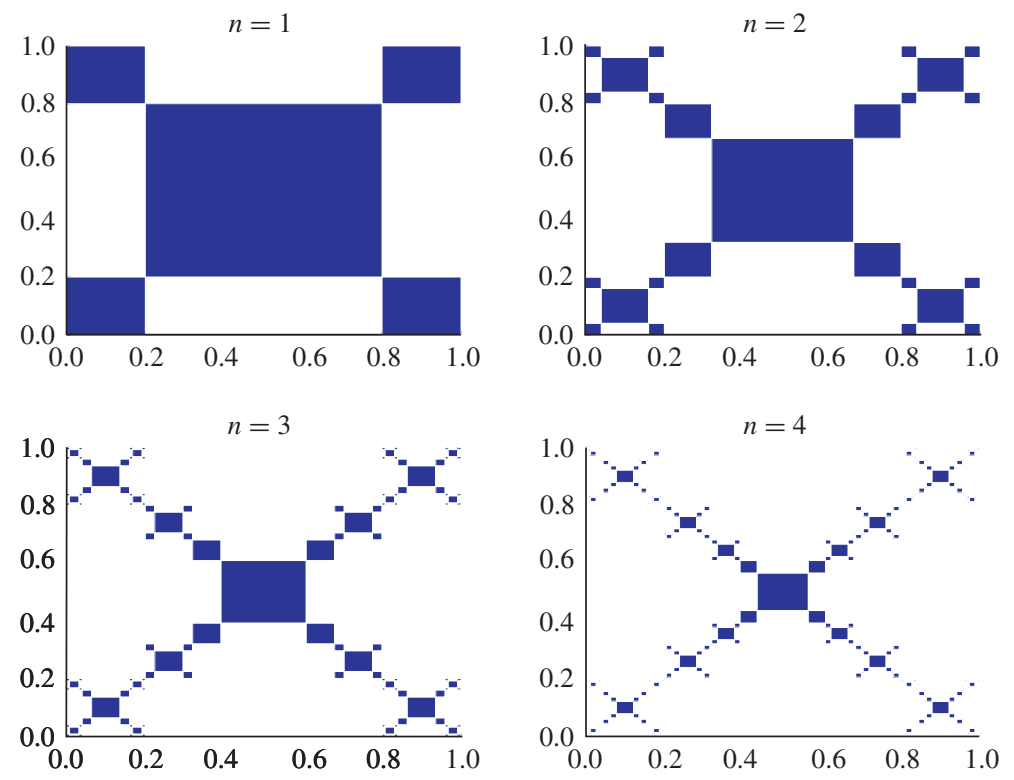

Figure 1: The support of $T^{n}(C)$, for $n=1,2,3,4$.

panel. This means that if $(U, V)$ has copula $T^{n}(C)$ for some $n \geq 1$, then $\left[(U, V) \mid U, V \leq t_{1}\right]$ has cumulative distribution function $T^{n-1}\left(C\left(x t_{1}, y t_{1}\right)\right), x, y \in[0,1]$. It follows that the copula of $\left[(U, V) \mid U, V \leq t_{1}\right]$ is exactly $T^{n-1}(C)$, i.e. $\Phi\left(T^{n}(C), t_{1}, t_{1}\right)=T^{n-1}(C)$. Using the same arguments, we find in general that $\Phi\left(T^{n}(C), t_{k}, t_{k}\right)=T^{n-k}(C)$. Finally, because $\Phi\left(\cdot, t_{k}, t_{k}\right)$ is continuous (see Lemma 4.3, below), it follows that

$$
\Phi\left(C_{T}, t_{k}, t_{k}\right)=\lim _{n \rightarrow \infty} \Phi\left(T^{n}(C), t_{k}, t_{k}\right)=\lim _{n \rightarrow \infty} T^{n-k}(C)=C_{T} .
$$

Remark 3.4. For $\alpha=\beta=1$, Theorem 3.1 generalizes Theorem 2.4 of Juri and Wüthrich (2004), which states that

$$
\lim _{u \rightarrow 0} \Phi(C, u, u)(x, y)=G\left(g^{-1}(x), g^{-1}(y)\right),
$$

where $g:[0, \infty) \rightarrow[0, \infty)$ is the strictly increasing, continuous function defined by $g(x):=$ $\lim _{u \rightarrow 0} C(x u, u) / C(u, u), G(x, y)=y^{\theta} g(x / y)$ for $(x, y) \in(0,1]^{2}$ (and vanishes elsewhere), and $\theta$ is a positive constant. In particular, Theorem 2.4 of Juri and Wüthrich (2004) applies to archimedean copulas having regularly varying generators, in which case the LTDC limit is the Clayton copula of Example 4.3, below, with parameter equal to minus the regular variation parameter (see Theorem 3.4 of Juri and Wüthrich (2004) and Theorem 3.3 of Juri and Wüthrich (2002)).

Remark 3.5. Following the previous remark, the analytical expression (3.5) for the limiting copula is due to the fact that homogeneous functions of order $\theta$ (in our case $G(x, y)=$ $\left.y^{\theta} g(x / y)\right)$ have closed-form expressions. Analogously, the closed-form expression (3.3) comes from the fact that generalized homogeneous functions such as $\phi$ in Theorem 3.1 also have closed-form representations (see the proof of Theorem 3.1, below, and Aczél (1966, p. 231) for more details). Unfortunately, this is not the case in higher dimensions; thus, assuming that 
Theorem 3.1 could be extended along the same lines to the multivariate case, the limiting copula would not have a closed-form expression (see Section 4.1).

Remark 3.6. There are many papers in the literature concerning multivariate extremes. In particular, bivariate extreme value distributions are obtained as limit laws of suitably normalized componentwise maxima; see, e.g. de Haan and Resnick (1977), Resnick (1987, Chapter 5), Coles and Tawn (1991), (1994), and Joe (1997, Chapter 6). It can be shown that the copula of any bivariate extreme value distribution satisfies the max-stability property, i.e.

$$
C^{t}(u, v)=C\left(u^{t}, v^{t}\right) \quad \text { for all }(u, v) \in[0,1]^{2} \text { and any } t>0 .
$$

As was mentioned in Juri and Wüthrich (2004), bivariate extreme value copulas differ from LTDC limits, the difference being similar to the one between the univariate generalized extreme value distributions and the generalized Pareto distribution. In fact, the generalized Pareto distribution lives on the log scale compared to generalized extreme value distributions (see Theorem 4.2 of Juri and Wüthrich (2004)). For instance, the Gumbel copula satisfies (3.6) but is not a LTDC limit. For a more detailed discussion about relations with other results from the area of multivariate extremes, we refer the reader to Juri and Wüthrich (2004).

We finish this section with an example of an LTDC limit that is not of the form (3.5). Below we will see that Theorem 4.1 provides a whole family of other examples of this type.

Proposition 3.1. Let $a, b:[0,1] \rightarrow[0,1]$ be two increasing functions, with $a(0)=b(0)=0$ and $a(1)=b(1)=1$, such that $t \mapsto a(t) / t$ and $t \mapsto b(t) / t$ are decreasing on $(0,1]$. Then

$$
C(x, y):=(a(x) y) \wedge(x b(y))
$$

defines a copula. Additionally, for $a \in \mathcal{R}_{\alpha}^{0}$ and $b \in \mathcal{R}_{\beta}^{0}$ with $(\alpha, \beta) \in[0,1]^{2} \backslash\{(0,0)\}$, and for directions $r$ and s such that

$$
\lim _{t \rightarrow 0} \frac{r(t) b(s(t))}{a(r(t)) s(t)}=1,
$$

we have

$$
\lim _{t \rightarrow 0} \Phi(C, r(t), s(t))(x, y)=\left(x^{\alpha} y\right) \wedge\left(x y^{\beta}\right),
$$

which is the Marshall and Olkin copula with parameters $1-\alpha$ and $1-\beta$.

Remark 3.7. Condition (3.8) is satisfied in the case in which $a(t)=t^{\alpha}, b(t)=t^{\beta}, r(t)=t^{\gamma}$, and $s(t)=t^{\delta}$, with $\beta \delta+\gamma=\alpha \gamma+\delta$.

\section{Invariant copulas}

There are many examples of (functional) limit theorems where the limit obtained is invariant under some kind of transformation. This is the case for the central limit theorem, where stable laws (which coincide with the class of possible limit laws for sums of independent, identically distributed random variables) are invariant under the sum operator. A similar result holds for the generalized extreme value distribution, which is the limit of maxima of independent, identically distributed random variables, as stated in the Fisher-Tippett theorem (Embrechts et al. (1997, Theorem 3.2.3)).

In our context, (2.7) can be seen as the result of a copula transformation mapping a copula, $C \in \mathcal{C}$, to its LTDC, $\Phi(C, u, v)$. Motivated by the above classical results, it therefore seems natural to look at copulas that are invariant under the LTDC transformation (2.7). 
Definition 4.1. We say that $C \in \mathcal{C}$ is invariant on the unit square if $\Phi(C, u, v)=C$ for all $(u, v) \in(0,1]^{2}$.

Lemma 4.1. Let $(U, V)$ have distribution function $C \in \mathcal{C}$, and let $(u, v) \in(0,1]^{2}$. Then, for $(x, y) \in[0, u] \times[0, v], \Phi(C, u, v)$ satisfies the identity

$$
\frac{C(x, y)}{C(u, v)}=\Phi(C, u, v)\left(\frac{C(x, v)}{C(u, v)}, \frac{C(u, y)}{C(u, v)}\right) .
$$

From Lemma 4.1, $C$ is invariant on the unit square if and only if, for any $(u, v) \in(0,1]^{2}$,

$$
\frac{C(x, y)}{C(u, v)}=C\left(\frac{C(x, v)}{C(u, v)}, \frac{C(u, y)}{C(u, v)}\right) \text { for all }(x, y) \in[0, u] \times[0, v] .
$$

A weaker type of invariance than that defined in Definition 4.1 is exemplified by copulas $C$ such that $\Phi(C, u, v)=C$ holds only for a particular set of parameters $(u, v) \in(0,1]^{2}$.

Definition 4.2. A copula $C \in \mathcal{C}$ is said to be invariant on the diagonal if $\Phi(C, u, u)=C$ for all $u \in(0,1]$. Similarly, $C \in \mathcal{C}$ is said to be invariant on the curve $\mathscr{D}=\{(r(t), s(t)): t \in T\}$, $T \subset \mathbb{R}$, where $r, s: T \rightarrow(0,1]$, whenever

$$
\Phi(C, r(t), s(t))=C \quad \text { for all } t \in T .
$$

Invariant copulas on the diagonal have been considered by Juri and Wüthrich (2002), (2004), and examples of such copulas are given in Examples 4.1 and 4.3, below.

Example 4.1. For $\alpha \in[0,1]$, consider the Cuadras-Augé copula

$$
C_{\alpha}(x, y):=\left(x^{1-\alpha} y\right) \wedge\left(x y^{1-\alpha}\right) .
$$

The copula $C_{\alpha}$ can be seen as a particular case of the Marshall and Olkin copula of Example 2.1 with identical parameters, and is a geometric mixture, with weights $\alpha$ and $1-\alpha$, of the upper Fréchet bound $C^{+}(x, y):=x \wedge y$ and the independent copula $C^{\perp}(x, y)=x y$. In fact,

$$
C_{\alpha}(x, y)=C^{+}(x, y)^{\alpha} C^{\perp}(x, y)^{1-\alpha} .
$$

For random variables $U$ and $V$ with joint distribution function $C_{\alpha}$, for $x$ and $y$ with $0 \leq x$, $y \leq u$ we have

$$
\begin{gathered}
F_{U}\left(C_{\alpha}, u, u\right)(x)=F_{V}\left(C_{\alpha}, u, u\right)(x)=\frac{C_{\alpha}(x, u)}{C_{\alpha}(u, u)}=\frac{x}{u}, \\
F\left(C_{\alpha}, u, u\right)(x, y)=\frac{C_{\alpha}(x, y)}{C_{\alpha}(u, u)}=C_{\alpha}\left(\frac{x}{u}, \frac{y}{u}\right) .
\end{gathered}
$$

Thus, from (2.7) we immediately find that $C_{\alpha}$ is an invariant copula on the diagonal.

A particular family of curve-invariant copulas is the one of Definition 4.3, below. We will see in Corollary 4.1 that this family of copulas coincides with the LTDC limits obtained in Theorem 3.1. 
Definition 4.3. Let $\alpha, \beta$, and $\theta$ be positive constants and let $P$ and $Q$ be increasing, continuous univariate distribution functions on $[0,1]$. We denote by $\mathscr{H}(\alpha, \beta, \theta)$ the set of two-dimensional distribution functions $H$ on $[0,1]^{2}$ that can be expressed as

$$
H(x, y)=x^{\theta / \alpha} h\left(y x^{-\beta / \alpha}\right), \quad \text { where } h(t):= \begin{cases}Q(t) & \text { if } t \in[0,1] \\ t^{\theta / \beta} P\left(t^{-\alpha / \beta}\right) & \text { if } t \in(1, \infty)\end{cases}
$$

Theorem 4.1. Let $\alpha, \beta, \theta>0$ and $H \in \mathscr{H}(\alpha, \beta, \theta)$. The copula relative to $H$ is given by

$$
\begin{aligned}
\Gamma(P, Q, \alpha, \beta, \theta)(u, v) \\
\quad:= \begin{cases}Q^{-1}(v)^{\theta / \beta} P\left(P^{-1}(u) Q^{-1}(v)^{-\alpha / \beta}\right) & \text { for } P^{-1}(u)^{\beta} \leq Q^{-1}(v)^{\alpha}, \\
P^{-1}(u)^{\theta / \alpha} Q\left(P^{-1}(u)^{-\beta / \alpha} Q^{-1}(v)\right) & \text { for } P^{-1}(u)^{\beta}>Q^{-1}(v)^{\alpha},\end{cases}
\end{aligned}
$$

and is invariant on $\mathcal{D}=\left\{\left(P\left(t^{\alpha}\right), Q\left(t^{\beta}\right)\right): t \in(0,1]\right\}$.

Remark 4.1. (a) From Theorem 4.1, we immediately obtain

$$
\lim _{t \rightarrow 0} \Phi\left(\Gamma(P, Q, \alpha, \beta, \theta), P\left(t^{\alpha}\right), Q\left(t^{\beta}\right)\right)=\Gamma(P, Q, \alpha, \beta, \theta)
$$

i.e. $\Gamma(P, Q, \alpha, \beta, \theta)$ is a LTDC limit. Furthermore, note that $\Gamma(g, g, 1,1, \theta)$ is precisely the copula in (3.5).

(b) Observe that $H$ in Theorem 4.1 is supposed to belong to $\mathscr{H}(\alpha, \beta, \theta)$, i.e. it is assumed that $H$ is a two-dimensional distribution function, whence $H$ has to satisfy the rectangle condition. If $H$ does not have a singular component then the rectangle condition can be checked by showing that the first-order and the mixed second-order derivatives of (6.6), below, are nonnegative. However, this need not always be the case for arbitrary choices of $P, Q, \alpha, \beta$, and $\theta$. This is the reason behind the hypothesis $H \in \mathscr{H}(\alpha, \beta, \theta)$ in Theorem 4.1: we assume that the conditions necessary for $H$ to be well defined are satisfied.

Example 4.2. The copula $\Gamma\left(\mathrm{Id}, \mathrm{Id}, \beta(\alpha+\beta-\alpha \beta)^{-1}, \alpha(\alpha+\beta-\alpha \beta)^{-1}, 1\right)$ is the Marshall and Olkin copula, which, by Theorem 4.1, is invariant on

$$
\mathscr{D}=\left\{\left(t^{\beta /(\alpha+\beta-\alpha \beta)}, t^{\alpha /(\alpha+\beta-\alpha \beta)}\right): t \in(0,1]\right\}=\left\{\left(t^{\beta}, t^{\alpha}\right): t \in(0,1]\right\} .
$$

Similarly, $\Gamma(\mathrm{Id}, \mathrm{Id}, \alpha, \beta, 1)$ is the Marshall and Olkin copula with parameters $(\alpha+\beta-1) / \alpha$ and $(\alpha+\beta-1) / \beta$.

Example 4.3. For $P(x)=2^{1 / \theta}\left(1+x^{-\theta}\right)^{-1 / \theta}$, the copula $\Gamma(P, P, \theta, \theta, \theta)$ is the Clayton copula with parameter $\theta$; i.e. for $(x, y) \in[0,1]^{2}$,

$$
\Gamma(P, P, \theta, \theta, \theta)(x, y)=\left(x^{-\theta}+y^{-\theta}-1\right)^{-1 / \theta} .
$$

By Theorem 4.1, this copula is invariant on $\mathcal{D}=\left\{\left(P\left(t^{\theta}\right), P\left(t^{\theta}\right)\right): t \in(0,1]\right\}=\left\{\left(t^{\theta}, t^{\theta}\right): t \in\right.$ $(0,1]\}$ for all $\theta$; i.e. $\Gamma(P, P, \theta, \theta, \theta)$ is invariant on $(0,1]^{2}$. The copula (4.3) is commonly referred to as the Clayton copula, although Clayton (1978) did not mention copulas and it was known before that paper appeared. The multivariate extension is sometimes attributed to Cook and Johnson (1981). 
Theorem 4.2 characterizes the possible LTDC limits, stating that they coincide with the set of invariant copulas on $(0,1]^{2}$. In particular, the family $\mathscr{H}(\alpha, \beta, \theta)$ characterizes LTDC limits on curves $\mathscr{D}=\{(r(t), s(t)): t \in T\}$, provided that the starting copula $C$ belongs to $\mathcal{C} \cap \mathcal{R}(r, s)$ and that $r$ and $s$ are strictly increasing, continuous, and regularly varying at 0 (see Corollary 4.1).

Theorem 4.2. If $C \in \mathcal{C}$ and $C_{0}$ are copulas such that $\lim _{u, v \rightarrow 0}\left\|\Phi(C, u, v)-C_{0}\right\|_{\infty}=0$, then $C_{0}$ is invariant on the unit square.

Corollary 4.1. Assume that $C$ satisfies the hypotheses of Theorem 3.1 and consider the copula $C_{0}=\lim _{t \rightarrow 0} \Phi(C, r(t), s(t))$. Then there is a constant, $\theta>0$, such that $C_{0}=\Gamma\left(\phi_{X}, \phi_{Y}, \alpha\right.$, $\beta, \theta)$ according to (4.2). As a consequence, $C_{0}$ is invariant on

$$
\mathscr{D}=\left\{\left(\phi_{X}\left(t^{\alpha}\right), \phi_{Y}\left(t^{\beta}\right)\right): t \in(0,1]\right\}
$$

The proof of Theorem 4.2 is based on the fact that $\Phi\left(C, u^{\prime}, v^{\prime}\right)$ can be seen as the LTDC obtained from another LTDC, $\Phi(C, u, v)$, with $u \geq u^{\prime}$ and $v \geq v^{\prime}$ (see Lemma 4.2). The second ingredient in the proof is the continuity of $\Phi(\cdot, u, v)$ (see Lemma 4.3). We state these preliminary results below and not just in the proofs in Section 6, since we believe them to be interesting in their own right.

Lemma 4.2. Let $C \in \mathcal{C}$. For $0 \leq u^{\prime} \leq u \leq 1$ and $0 \leq v^{\prime} \leq v \leq 1$ we have

(i) $\Phi\left(C, u^{\prime}, v^{\prime}\right)=\Phi\left(\Phi(C, u, v), u^{*}, v^{*}\right)$, where $u^{*}=C\left(u^{\prime}, v\right) / C(u, v)$ and $v^{*}=$ $C\left(u, v^{\prime}\right) / C(u, v)$, and

(ii) $\Phi\left(\Phi(C, u, v), u^{\prime}, v^{\prime}\right)=\Phi\left(C, u^{*}, v^{*}\right)$, where $u^{*}$ and $v^{*}$ satisfy the relations $C\left(u^{*}, v\right)=$ $u^{\prime} C(u, v)$ and $C\left(u, v^{*}\right)=v^{\prime} C(u, v)$, respectively.

Lemma 4.3. For any $u, v \in(0,1]$, the map $\mathcal{C} \rightarrow \mathcal{C}$ given by $C \mapsto \Phi(C, u, v)$ is continuous with respect to the $\|\cdot\|_{\infty}$-norm.

Remark 4.2. The parameters $\alpha$ and $\beta$ of the LTDC limit $\Gamma(P, Q, \alpha, \beta, \theta)$ can be interpreted as parameters describing the direction under which the limit is taken, since, as stated in Theorem 4.1, $\Gamma(P, Q, \alpha, \beta, \theta)$ is invariant on $\mathscr{D}=\left\{\left(P\left(t^{\alpha}\right), Q\left(t^{\beta}\right)\right): t \in(0,1]\right\}$. However, such a distribution is not identifiable. In fact, $\alpha, \beta$, and $\theta$ are defined up to a positive multiplicative constant; thus, $\Gamma(P, Q, \alpha, \beta, \theta)$ could be defined using two parameters only. More precisely, for $\eta:=\beta / \alpha$,

$$
\Gamma(P, Q, \alpha, \beta, \theta)=\Gamma(P, Q, 1, \eta, \theta)=: \Gamma(P, Q, \eta, \theta) .
$$

Moreover, for all $k>0$ we have

$$
\Gamma(P, Q, \eta, \theta)=\Gamma\left(P_{k}, Q_{k}, k \eta, k \theta\right),
$$

where $P_{k}(x):=P\left(x^{k}\right)$ and $Q_{k}(x):=Q\left(x^{k}\right), x \in[0,1]$.

We finish this section with a proposition.

Proposition 4.1. The only copula that is absolutely continuous and invariant on $[0,1]^{2}$ is the Clayton copula. 


\subsection{Multivariate extensions}

All the results obtained so far have been formulated in the bivariate case. However, observe that the concept of an LTDC can easily be extended to higher dimensions. Let $\boldsymbol{U}=$ $\left(U_{1}, \ldots, U_{n}\right)$ be a random vector with standard uniform marginals such that its distribution function (copula), $C$, is strictly increasing in all its components. Then, for any $\boldsymbol{u}=\left(u_{1}, \ldots, u_{n}\right) \in$ $(0,1]^{n}$, consider the distribution function $F_{i \mid \boldsymbol{u}}$ of $U_{i}$ given the event $\left\{U_{1} \leq u_{1}, \ldots, U_{n} \leq u_{n}\right\}$ (written $\{\boldsymbol{U} \leq \boldsymbol{u}\}$ ), which is given by

$$
F_{i \mid \boldsymbol{u}}\left(x_{i}\right)=\frac{C\left(u_{1}, \ldots, u_{i-1}, x_{i}, u_{i+1}, \ldots, u_{n}\right)}{C\left(u_{1}, \ldots, u_{i-1}, u_{i}, u_{i+1}, \ldots, u_{n}\right)},
$$

whence the copula of $\boldsymbol{U}$ given $\{\boldsymbol{U} \leq \boldsymbol{u}\}$ is

$$
\Phi\left(C, u_{1}, \ldots, u_{n}\right)\left(x_{1}, \ldots, x_{n}\right):=\frac{C\left(F_{1 \mid \boldsymbol{u}}^{-1}\left(x_{1}\right), \ldots, F_{n \mid \boldsymbol{u}}^{-1}\left(x_{n}\right)\right)}{C\left(u_{1}, \ldots, u_{n}\right)} .
$$

In this setup, it can be easily shown that Proposition 4.1 also holds in higher dimensions; i.e. that the only absolutely continuous copula which is invariant on the unit cube is the Clayton copula. The proof of this generalized result is almost the same as that in the bivariate case: differentiating with respect to $u_{i}$ and $u_{j}$ yields the equation analogous to (6.20), for some parameter $\theta_{i j}$, and symmetry arguments can be used to show that $\theta_{i j}$ does not depend on $i$ or $j$ and, therefore, that $\theta_{i j}=\theta$. In addition, note that the upper Fréchet bound is also invariant, but does not satisfy the assumption of absolute continuity. Actually, we conjecture that it is the only non-absolutely continuous copula which is invariant.

Results for limiting copulas in the $n$-dimensional case $(n \geq 2)$ can be obtained by the arguments used in Section 3, with Definition 3.2 generalized appropriately for functions $r_{1}, \ldots, r_{n}$ and $\phi$. More precisely, assuming that the $r_{i}$ are regularly varying with $r_{i} \in \mathcal{R}_{\alpha_{i}}^{0}$ for some $\alpha_{i}$, it can be shown that a limiting function $\phi$ exists and that Theorem (2.1) of de Haan et al. (1984) still holds. In other words, $\phi$ is necessarily a generalized homogeneous function; i.e. there exists a $\theta \in \mathbb{R}$ such that, for all $x_{1}, \ldots, x_{n}, t$,

$$
\phi\left(t^{\alpha_{1}} x_{1}, t^{\alpha_{2}} x_{2}, \ldots, t^{\alpha_{n}} x_{n}\right)=t^{\theta} \phi\left(x_{1}, x_{2}, \ldots, x_{n}\right) .
$$

Unfortunately, an explicit expression for $\phi$ can be derived only for $n=2$.

\section{An application to credit risk}

The main risk drivers of almost all credit derivatives such as, e.g. credit default swap baskets or first-to-default contract types are given by the relevant default times. Among the most popular (univariate) default time models are those that are intensity based. As was shown by Schönbucher and Schubert (2001), a copula approach allows us to naturally model arbitrary dependence structures in such an intensity-based framework.

In this section we first review the setup of Schönbucher and Schubert (2001), and then show how our LTDC limits can be used as dependence structures for credit stress scenarios.

\subsection{Intensity-based default models}

For $\sigma$-algebras $\mathcal{A}$ and $\mathcal{B}$ with $\mathcal{A} \subset \mathcal{B}$, and for a set $B \in \mathcal{B}$, in the sequel we will use the notation $\mathcal{A} \wedge B:=\{A \cap B: A \in \mathcal{A}\}$. Furthermore, all filtrations are supposed to satisfy the usual conditions. Finally, for a review of point process intensities, we refer the reader to Brémaud (1981). 
Schönbucher and Schubert (2001) proposed the following intensity-based default model, which we here recall in the two-dimensional case. Let $\lambda_{i}, i=1,2$, be nonnegative càdlàg processes adapted to a filtration, $\left(g_{t}\right)_{t \geq 0}$, representing general market information other than explicit information on the occurrence of defaults. For standard, uniformly distributed random variables $U_{1}$ and $U_{2}$, which are assumed to be independent of $g_{\infty}:=\bigcup_{t \geq 0} g_{t}$, we define the default times as the random variables

$$
\tau_{i}:=\inf \left\{t>0: \gamma_{i}(t) \leq U_{i}\right\}, \quad i=1,2,
$$

where the $\gamma_{i}(t):=\exp \left(-\Lambda_{i}(t)\right)$ are called countdown processes, with $\Lambda_{i}(t):=\int_{0}^{t} \lambda_{i}(s) \mathrm{d} s$. Note that, conditioned on $g_{\infty}$, we have

$$
\mathrm{P}\left[\tau_{1} \leq t_{1}, \tau_{2} \leq t_{2} \mid g_{\infty}\right]=\hat{C}\left(\gamma_{1}\left(t_{1}\right), \gamma_{2}\left(t_{2}\right)\right),
$$

where $C$ is the distribution function of $\left(U_{1}, U_{2}\right)$. Thus, we see that by defining default times as in (5.1), given general market information the default dependence mechanism is completely described by $C$.

Remark 5.1. The motivation behind (5.1) comes from the fact that, for a Cox process with intensity $\lambda$, the time, $\tau$, of the first jump can be written as

$$
\tau=\inf \left\{t>0: \int_{0}^{t} \lambda(s) \mathrm{d} s \geq Z\right\},
$$

where $Z$ is exponentially distributed with parameter 1 (see Lando (1998)).

In general, the intensity of a point process depends on the information upon which it is conditioned. Denoting by $N_{i}$ the default counting process of counterparty $i=1,2$, and by $\mathcal{F}_{t}^{i}$ the augmented filtration of $\sigma\left(N_{i}(s): 0 \leq s \leq t\right)$, we find that $\lambda_{i}$ is the $\mathcal{F}_{t}^{i}$-intensity of $N_{i}$. However, it is in the spirit of any multivariate model also to consider the information relative to the other counterparties, such as the one given by $C$ and $\mathscr{H}_{t}:=\bigvee_{i=1,2}\left(\mathcal{F}_{t}^{i} \vee g_{t}\right), t \geq 0$. Indeed, we find from Schönbucher and Schubert (2001) that the $\mathscr{H}_{t}$-intensity, $h_{i}$, of $N_{i}$ equals

$$
h_{i}(t):=\lambda_{i}(t) \gamma_{i}(t) \partial_{i} \log \left(C\left(\gamma_{1}(t), \gamma_{2}(t)\right)\right) .
$$

Because of the term $\partial_{i} \log \left(C\left(\gamma_{1}(t), \gamma_{2}(t)\right)\right)$, the intensity of a single counterparty is also affected by the dependence structure of the several counterparties. In the case in which $U_{1}$ and $U_{2}$ are independent, i.e. whenever $C=C^{\perp}$, the right-hand side of (5.3) reduces to $\lambda_{i}(t)$, i.e. to the $\mathcal{F}_{t}^{i}$-intensity of $N_{i}$. Furthermore, with the additional information that the other obligor has already defaulted, i.e. $\left\{\tau_{j}=t_{j}\right\}, j \neq i, t_{j}>0$, the default intensity of the surviving counterparty takes the form

$$
h_{i}^{-j}(t):=\lambda_{i}(t) \gamma_{i}(t) \frac{\partial_{i j} C\left(\gamma_{1}(t), \gamma_{2}(t)\right)}{\partial_{j} C\left(\gamma_{1}(t), \gamma_{2}(t)\right)} .
$$

A special case of (5.3) and (5.4) is when $C$ equals the Clayton copula with parameter $\theta$, of Example 4.3. In that case,

$$
h_{i}(t)=\left(\frac{C\left(\gamma_{1}(t), \gamma_{2}(t)\right)}{\gamma_{i}(t)}\right)^{\theta} \lambda_{i}(t) \text { and } h_{i}^{-j}(t)=(1+\theta) h_{i}(t) .
$$

As stated in Schönbucher and Schubert (2001), such a dependence structure reflects one of the main features of a model introduced by Davis and Lo (2001a), (2001b), where knowledge of one obligor's default determines a jump in the spread of the other obligor by a factor of $1+\theta$. 


\subsection{Dependence structures for stress scenarios}

Stress scenarios for default times arise in many different situations. For example, pension funds have to invest only in investment-grade bonds for regulatory reasons. Thus, a default (or downgrade) of a bond in the pension fund's portfolio determines the replacement of that bond, possibly resulting in (large) losses due to the bond's value decrease. Another example is given by first-to-default credit default swap baskets, where, in the case of an 'early' default, the protection seller receives the premium only for a short time but has to deliver the notional very soon.

More generally, knowing or modeling the dependence structure of the various default times, and in particular the joint behavior under adverse market conditions, avoids risk underestimation, allowing for risk-adjusted pricing (e.g. of credit derivatives). Such stress situations can be described by conditional distributions of the type

$$
\mathrm{P}\left[\tau_{1} \leq t_{1}, \tau_{2} \leq t_{2} \mid g_{\infty} \wedge\left\{\tau_{1} \leq T, \tau_{2} \leq T\right\}\right]
$$

as $T$ tends to 0 . Since the conditional distribution of $\tau_{i}$ given $\mathcal{H}_{t}^{i}$ equals $\gamma_{i}(t)$, it follows from Proposition 2.1 and (5.2) that the copula relative to the conditional distribution in (5.5) is given by $\Phi\left(\hat{C}, 1-\gamma_{1}(T), 1-\gamma_{2}(T)\right)$, where $\hat{C}$ is the survival copula of $C$.

Example 5.1. (First-to-default time.) The conditional distribution of the first-to-default time $\tau:=\tau_{1} \wedge \tau_{2}$ conditioned on $g_{\infty} \wedge\left\{\tau_{1} \leq T, \tau_{2} \leq T\right\}$ is given, for $t \leq T$, by

$$
\begin{aligned}
\mathrm{P}\left[\tau \leq t \mid g_{\infty} \wedge\left\{\tau_{1} \leq T, \tau_{2} \leq T\right\}\right] & =1-\mathrm{P}\left[\tau_{1}>t, \tau_{2}>t \mid g_{\infty} \wedge\left\{\tau_{1} \leq T, \tau_{2} \leq T\right\}\right] \\
& =1-C^{*}\left(1-\gamma_{1}(t), 1-\gamma_{2}(t)\right),
\end{aligned}
$$

where $C^{*}$ is the survival copula of $\Phi\left(\hat{C}, 1-\gamma_{1}(T), 1-\gamma_{2}(T)\right)$.

Suppose now that $\lambda_{i}$ is regularly varying at 0 with parameter $\delta_{i} \geq 0$, which, as is easy to check, implies that $1-\gamma_{i} \in \mathcal{R}_{1+\delta_{i}}^{0}$. Furthermore, assume that $\hat{C} \in \mathcal{C} \cap \mathcal{R}\left(1-\gamma_{1}, 1-\gamma_{2}\right)$ with limiting function $\phi$. Then, from Corollary 4.1, there is a constant, $\theta>0$, such that

$$
\lim _{T \rightarrow 0} \Phi\left(\hat{C}, 1-\gamma_{1}(T), 1-\gamma_{2}(T)\right)=\Gamma\left(\phi_{X}, \phi_{Y}, 1+\delta_{1}, 1+\delta_{2}, \theta\right) .
$$

As a special case, for $\gamma_{1}=\gamma_{2}=: \gamma$ and $\delta_{1}=\delta_{2}=0$ we have

$$
\lim _{T \rightarrow 0} \Phi(\hat{C}, 1-\gamma(T), 1-\gamma(T))=\Gamma(g, g, 1,1, \theta), \quad g:=\phi_{X},
$$

which corresponds to the limiting copula (3.5).

As we mentioned at the end of Section 3, a special case of Theorem 3.1 is when the starting copula is archimedean with a regularly varying generator. In this case, the LTDC limit on the diagonal is the Clayton copula. Thus, the Davis-Lo model can be seen as a stress-scenario model.

\section{Proofs}

Proof of Proposition 2.1. For $w_{i}:=G_{i}\left(z_{i}\right), i=1,2$, we have, by definition,

$$
\Phi\left(C, G_{1}\left(z_{1}\right), G_{2}\left(z_{2}\right)\right)\left(u_{1}, u_{2}\right)=\frac{C\left(F_{U_{1}}\left(C, w_{1}, w_{2}\right)^{-1}\left(u_{1}\right), F_{U_{2}}\left(C, w_{1}, w_{2}\right)^{-1}\left(u_{2}\right)\right)}{C\left(G_{1}\left(z_{1}\right), G_{2}\left(z_{2}\right)\right)} .
$$


Furthermore,

$$
\begin{aligned}
F_{U_{1}}\left(C, w_{1}, w_{2}\right)\left(v_{1}\right) & =\frac{C\left(v_{1}, w_{2}\right)}{C\left(w_{1}, w_{2}\right)}=\frac{C\left(v_{1}, G_{2}\left(z_{2}\right)\right)}{C\left(G_{1}\left(z_{1}\right), G_{2}\left(z_{2}\right)\right)}=\frac{G\left(G_{1}^{-1}\left(v_{1}\right), z_{2}\right)}{G\left(z_{1}, z_{2}\right)} \\
& =G_{1}^{z_{1}, z_{2}}\left(G_{1}^{-1}\left(v_{1}\right)\right),
\end{aligned}
$$

whence $F_{U_{1}}\left(C, w_{1}, w_{2}\right)^{-1}\left(u_{1}\right)=G_{1}\left(\left(G_{1}^{z_{1}, z_{2}}\right)^{-1}\left(u_{1}\right)\right)$. Similarly, $F_{U_{2}}\left(C, w_{1}, w_{2}\right)^{-1}\left(u_{2}\right)=$ $G_{2}\left(\left(G_{2}^{z_{1}, z_{2}}\right)^{-1}\left(u_{2}\right)\right)$. Thus,

$$
\begin{aligned}
\Phi\left(C, G_{1}\left(z_{1}\right), G_{2}\left(z_{2}\right)\right)\left(u_{1}, u_{2}\right) & =\frac{C\left(G_{1}\left(\left(G_{1}^{z_{1}, z_{2}}\right)^{-1}\left(u_{1}\right)\right), G_{2}\left(\left(G_{2}^{z_{1}, z_{2}}\right)^{-1}\left(u_{2}\right)\right)\right)}{G\left(z_{1}, z_{2}\right)} \\
& =\frac{G\left(\left(G_{1}^{z_{1}, z_{2}}\right)^{-1}\left(u_{1}\right),\left(G_{2}^{z_{1}, z_{2}}\right)^{-1}\left(u_{2}\right)\right)}{G\left(z_{1}, z_{2}\right)} \\
& =G^{z_{1}, z_{2}}\left(\left(G_{1}^{z_{1}, z_{2}}\right)^{-1}\left(u_{1}\right),\left(G_{2}^{z_{1}, z_{2}}\right)^{-1}\left(u_{2}\right)\right) \\
& =\Phi\left(G, z_{1}, z_{2}\right)\left(u_{1}, u_{2}\right) .
\end{aligned}
$$

Proof of Theorem 3.1. The proof of this theorem is based on the following lemma, whose proof is straightforward and is therefore left to the reader.

Lemma 6.1. Suppose that the random vectors $\left(X_{n}, Y_{n}\right)$ have continuous, strictly increasing marginals and are such that $\lim _{n \rightarrow \infty}\left(X_{n}, Y_{n}\right)=(X, Y)$ in distribution for some $(X, Y)$. Then

$$
\lim _{n \rightarrow \infty}\left\|C_{n}-C\right\|_{\infty}=0
$$

where $C_{n}$ and $C$ denote the copulas of $\left(X_{n}, Y_{n}\right)$ and $(X, Y)$, respectively.

Now let $(U, V)$ have distribution function $C$. Note that

$$
\begin{aligned}
\frac{C(r(t) x, s(t))}{C(r(t), s(t))} & =\mathrm{P}[U \leq r(t) x \mid U \leq r(t), V \leq s(t)], \\
\frac{C(r(t), s(t) y)}{C(r(t), s(t))} & =\mathrm{P}[V \leq s(t) y \mid U \leq r(t), V \leq s(t)], \\
\frac{C(r(t) x, s(t) y)}{C(r(t), s(t))} & =\mathrm{P}[U \leq r(t) x, V \leq s(t) y \mid U \leq r(t), V \leq s(t)] ;
\end{aligned}
$$

i.e. the distributions in (6.1)-(6.3) are respectively the conditional distributions of $U / r(t)$, $V / s(t)$, and $(U / r(t), V / s(t))$, given that $U \leq r(t)$ and $V \leq s(t)$. Since copulas are invariant under strictly increasing transformations of the underlying variables, it follows that we can use the conditional distributions in (6.1)-(6.3), instead of $F_{U}(C, r(t), s(t)), F_{V}(C, r(t), s(t))$, and $F(C, r(t), s(t))$, to construct $\Phi(C, r(t), s(t))$. Furthermore, since $C \in \mathcal{C}$ and because $r$ and $s$ are strictly increasing and continuous, it follows that the distributions in (6.1)-(6.3) are also continuous and strictly increasing. By hypothesis, we have $C \in \mathcal{R}(r, s)$, i.e.

$$
\lim _{t \rightarrow 0} \frac{C(r(t) x, s(t) y)}{C(r(t), s(t))}=\phi(x, y) \quad \text { for all } x, y \in[0,1],
$$

implying that the expressions in (6.1)-(6.3) respectively converge to $\phi_{X}, \phi_{Y}$, and $\phi$, as $t \rightarrow 0$. Thus, by applying Lemma 6.1, we obtain

$$
\lim _{t \rightarrow 0} \Phi(C, r(t), s(t))(x, y)=\phi\left(\phi_{X}^{-1}(x), \phi_{Y}^{-1}(y)\right),
$$


from which (3.2) follows. Since $r \in \mathcal{R}_{\alpha}^{0}$ and $s \in \mathcal{R}_{\beta}^{0}$, Theorem 2.1 of de Haan et al. (1984) implies that there is a $\theta>0$ such that, for all $t, x, y>0$,

$$
\phi\left(t^{\alpha} x, t^{\beta} y\right)=t^{\theta} \phi(x, y) .
$$

Furthermore, according to Aczél (1966, p. 231) the most general solution to the functional equation (6.4) is given by

$$
\phi(x, y)= \begin{cases}x^{\theta / \alpha} h\left(y x^{-\beta / \alpha}\right) & \text { if } x \neq 0, \\ c y^{\theta / \beta} & \text { if } x=0 \text { and } y \neq 0, \\ 0 & \text { if } x=y=0,\end{cases}
$$

where $c$ is a constant and $h$ is a function of one variable. Because $\phi(0, y)=0$ and $\phi_{Y}(y)=$ $\phi(1, y)=h(y)$, it follows that $c=0$ and that the restriction of $h$ to [0,1] equals $\phi_{Y}$. Furthermore, for $x \in(0,1]$ we have

$$
\phi_{X}\left(x^{\alpha / \beta}\right)=\phi\left(x^{\alpha / \beta}, 1\right)=x^{\theta / \beta} h(1 / x),
$$

whence, for $t=1 / x>1$, we obtain $h(t)=h(1 / x)=x^{-\theta / \beta} \phi_{X}\left(x^{\alpha / \beta}\right)=t^{\theta / \beta} \phi_{X}\left(t^{-\alpha / \beta}\right)$, from which (3.3) and, therefore, the proof of Theorem 3.1 follow.

Proof of Proposition 3.1. In order to prove that (3.7) defines a copula, we have to prove (2.1) and (2.2). For $x \in[0,1]$, the conditions $C(x, 0)=C(0, x)=0$ are satisfied because $a(0)=b(0)=0$. Furthermore, since $x \mapsto a(x) / x$ is decreasing, with $a(1)=1$, we have $a(x) \geq x$ for any $x \in[0,1]$. Thus, because $b(1)=1$, we obtain $C(x, 1)=a(x) \wedge x=x$. Similarly, $C(1, y)=y$ for $y \in[0,1]$, which proves (2.1). Now, for $0<x_{1} \leq x_{2} \leq 1$ and $0<y_{1} \leq y_{2} \leq 1$

$$
\begin{aligned}
\Delta:= & C\left(x_{2}, y_{2}\right)-C\left(x_{1}, y_{2}\right)-C\left(x_{2}, y_{1}\right)+C\left(x_{1}, y_{1}\right) \\
= & x_{2} y_{2}\left(\frac{a\left(x_{2}\right)}{x_{2}} \wedge \frac{b\left(y_{2}\right)}{y_{2}}\right)-x_{1} y_{2}\left(\frac{a\left(x_{1}\right)}{x_{1}} \wedge \frac{b\left(y_{2}\right)}{y_{2}}\right)-x_{2} y_{1}\left(\frac{a\left(x_{2}\right)}{x_{2}} \wedge \frac{b\left(y_{1}\right)}{y_{1}}\right) \\
& +x_{1} y_{1}\left(\frac{a\left(x_{1}\right)}{x_{1}} \wedge \frac{b\left(y_{1}\right)}{y_{1}}\right) .
\end{aligned}
$$

Since $x \mapsto a(x) / x$ and $x \mapsto b(x) / x$ are decreasing, six different cases have to be considered.

(i) If $a\left(x_{2}\right) / x_{2} \leq a\left(x_{1}\right) / x_{1} \leq b\left(y_{2}\right) / y_{2} \leq b\left(y_{1}\right) / y_{1}$ then

$$
\Delta=\left(y_{2}-y_{1}\right)\left(a\left(x_{2}\right)-a\left(x_{1}\right)\right) \geq 0,
$$

since $a$ is increasing.

(ii) If $b\left(y_{2}\right) / y_{2} \leq b\left(y_{1}\right) / y_{1} \leq a\left(x_{2}\right) / x_{2} \leq a\left(x_{1}\right) / x_{1}$ then

$$
\Delta=\left(x_{2}-x_{1}\right)\left(b\left(y_{2}\right)-b\left(y_{1}\right)\right) \text {, }
$$

which is nonnegative.

(iii) If $a\left(x_{2}\right) / x_{2} \leq b\left(y_{2}\right) / y_{2} \leq b\left(y_{1}\right) / y_{1} \leq a\left(x_{1}\right) / x_{1}$ then

$$
\Delta=x_{1}\left(b\left(y_{1}\right)-b\left(y_{2}\right)\right)+a\left(x_{2}\right)\left(y_{2}-y_{1}\right),
$$


which is nonnegative if and only if

$$
\frac{b\left(y_{2}\right)-b\left(y_{1}\right)}{y_{2}-y_{1}} \leq \frac{a\left(x_{2}\right)}{x_{1}} .
$$

Since $x \mapsto b(x) / x$ is decreasing, the left-hand side of (6.5) can be bounded as follows:

$$
\frac{b\left(y_{2}\right)-b\left(y_{1}\right)}{y_{2}-y_{1}}=\frac{b\left(y_{2}\right)}{y_{2}} \frac{y_{2}}{y_{2}-y_{1}}-\frac{b\left(y_{1}\right)}{y_{1}} \frac{y_{1}}{y_{2}-y_{1}} \leq \frac{b\left(y_{1}\right)}{y_{1}} .
$$

By hypothesis and since $a$ is increasing, we have $b\left(y_{1}\right) / y_{1} \leq a\left(x_{1}\right) / x_{1} \leq a\left(x_{2}\right) / x_{1}$, from which (6.5) follows.

(iv) If $b\left(y_{2}\right) / y_{2} \leq a\left(x_{2}\right) / x_{2} \leq a\left(x_{1}\right) / x_{1} \leq b\left(y_{1}\right) / y_{1}$ then

$$
\Delta=\left(a\left(x_{1}\right)-a\left(x_{2}\right)\right) y_{1}+\left(x_{2}-x_{1}\right) b\left(y_{2}\right),
$$

which can be shown to be nonnegative using the same arguments as in (iii).

(v) If $a\left(x_{2}\right) / x_{2} \leq b\left(y_{2}\right) / y_{2} \leq a\left(x_{1}\right) / x_{1} \leq b\left(y_{1}\right) / y_{1}$ then

$$
\Delta=\left(y_{2}-y_{1}\right) a\left(x_{2}\right)-x_{1} b\left(y_{2}\right)+y_{1} a\left(x_{1}\right) .
$$

By hypothesis,

$$
x_{1} b\left(y_{2}\right) \leq a\left(x_{1}\right) y_{2}=a\left(x_{1}\right) y_{1}+a\left(x_{1}\right)\left(y_{2}-y_{1}\right) \leq a\left(x_{1}\right) y_{1}+a\left(x_{2}\right)\left(y_{2}-y_{1}\right),
$$

where the last inequality follows because $a$ is increasing. This shows that $\Delta \geq 0$.

(vi) If $b\left(y_{2}\right) / y_{2} \leq a\left(x_{2}\right) / x_{2} \leq b\left(y_{1}\right) / y_{1} \leq a\left(x_{1}\right) / x_{1}$ then

$$
\Delta=\left(x_{2}-x_{1}\right) b\left(y_{2}\right)-y_{1} a\left(x_{2}\right)+x_{1} b\left(y_{1}\right) .
$$

As in (v), it follows that $\Delta \geq 0$.

To prove (3.9), consider

$$
\begin{aligned}
\frac{C(r(t) x, s(t) y)}{C(r(t), s(t))}= & \frac{[a(r(t) x) s(t) y] \wedge[r(t) x b(s(t) y)]}{[a(r(t)) s(t)] \wedge[r(t) b(s(t))]} \\
= & \left(\left[\frac{a(r(t) x)}{a(r(t))} y\right] \wedge\left[x \frac{b(s(t) y)}{b(s(t))} \frac{r(t)}{a(r(t))} \frac{b(s(t))}{s(t)}\right]\right) \\
& \times\left(1 \wedge\left[\frac{r(t)}{a(r(t))} \frac{b(s(t))}{s(t)}\right]\right)^{-1} .
\end{aligned}
$$

Since, by hypothesis, $a \in \mathcal{R}_{\alpha}^{0}, b \in \mathcal{R}_{\beta}^{0}$, and $\lim _{t \rightarrow 0} r(t) b(s(t)) /(a(r(t)) s(t))=1$, it follows that

$$
\lim _{t \rightarrow 0} \frac{C(r(t) x, s(t) y)}{C(r(t), s(t))}=\left(x^{\alpha} y\right) \wedge\left(x y^{\beta}\right)=: \phi(x, y) ;
$$

i.e. $C \in \mathcal{C} \cap \mathcal{R}(r, s)$ with limiting function $\phi$. Since $0<\alpha, \beta \leq 1$, we have $\phi_{X}(x)=\phi(x, 1)=$ $x$ and $\phi_{Y}(y)=\phi(1, y)=y$, whence, from Theorem 3.1,

$$
\lim _{t \rightarrow 0} \Phi(C, r(t), s(t))(x, y)=\phi\left(\phi_{X}^{-1}(x), \phi_{Y}^{-1}(y)\right)=\left(x^{\alpha} y\right) \wedge\left(x y^{\beta}\right) .
$$


Proof of Lemma 4.1. Because $C \in \mathcal{C}, F_{U}(C, u, v)$ and $F_{V}(C, u, v)$ are strictly increasing. From Sklar's theorem and (2.5) and (2.6), we obtain

$$
\begin{aligned}
\Phi(C, u, v)\left(\frac{C(x, v)}{C(u, v)}, \frac{C(u, y)}{C(u, v)}\right) & =\Phi(C, u, v)\left(F_{U}(C, u, v)(x), F_{V}(C, u, v)(y)\right) \\
& =F(C, u, v)(x, y) \\
& =\frac{C(x, y)}{C(u, v)} .
\end{aligned}
$$

This completes the proof of Lemma 4.1.

Proof of Theorem 4.1. We will first prove that $\Gamma(P, Q, \alpha, \beta, \theta)$ defined by (4.2) is a copula and then show the invariance property. The function $H$ defined by (4.1) can be rewritten as

$$
\begin{aligned}
H(x, y) & = \begin{cases}x^{\theta / \alpha}\left[y x^{-\beta / \alpha}\right]^{\theta / \beta} P\left(\left[y x^{-\beta / \alpha}\right]^{-\alpha / \beta}\right) & \text { if } x^{\beta}<y^{\alpha}, \\
x^{\theta / \alpha} Q\left(y x^{-\beta / \alpha}\right) & \text { if } x^{\beta} \geq y^{\alpha},\end{cases} \\
& = \begin{cases}y^{\theta / \beta} P\left(y^{-\alpha / \beta} x\right) & \text { if } x^{\beta}<y^{\alpha}, \\
x^{\theta / \alpha} Q\left(y x^{-\beta / \alpha}\right) & \text { if } x^{\beta} \geq y^{\alpha} .\end{cases}
\end{aligned}
$$

By hypothesis, the marginals, $P$ and $Q$, of $H$ are strictly increasing, continuous functions. It thus follows from Sklar's theorem that the copula associated with $H$ is

$$
H\left(P^{-1}(u), Q^{-1}(v)\right)= \begin{cases}Q^{-1}(v)^{\theta / \beta} P\left(P^{-1}(u) Q^{-1}(v)^{-\alpha / \beta}\right) & \text { if } P^{-1}(u)^{\beta}<Q^{-1}(v)^{\alpha}, \\ P^{-1}(u)^{\theta / \alpha} Q\left(P^{-1}(u)^{-\beta / \alpha} Q^{-1}(v)\right) & \text { if } P^{-1}(u)^{\beta} \geq Q^{-1}(v)^{\alpha},\end{cases}
$$

which is precisely $\Gamma(P, Q, \alpha, \beta, \theta)$. We now show that $\Gamma(P, Q, \alpha, \beta, \theta)$ is invariant on the curve $\mathcal{D}=\left\{\left(P\left(t^{\alpha}\right), Q\left(t^{\beta}\right)\right): t \in(0,1]\right\}$. For notational convenience we denote $\Gamma(P, Q, \alpha$, $\beta, \theta$ ) by $C$. To derive the LTDC associated with $C$, we first note that, from (6.7),

$$
\begin{aligned}
C\left(P\left(t^{\alpha}\right), Q\left(t^{\beta}\right)\right) & =t^{\theta}, \\
C\left(x, Q\left(t^{\beta}\right)\right) & = \begin{cases}t^{\theta} P\left(P^{-1}(x) t^{-\alpha}\right) & \text { if } P^{-1}(x)<t^{\alpha}, \\
P^{-1}(x)^{\theta / \alpha} Q\left(P^{-1}(x)^{-\beta / \alpha} t^{\beta}\right) & \text { if } P^{-1}(x) \geq t^{\alpha},\end{cases} \\
C\left(P\left(t^{\alpha}\right), y\right) & = \begin{cases}Q^{-1}(y)^{\theta / \beta} P\left(t^{\alpha} Q^{-1}(y)^{-\alpha / \beta}\right) & \text { if } t^{\beta}<Q^{-1}(y), \\
t^{\theta} Q\left(t^{-\beta} Q^{-1}(y)\right) & \text { if } t^{\beta} \geq Q^{-1}(y) .\end{cases}
\end{aligned}
$$

Now let $(x, y) \in\left[0, P\left(t^{\alpha}\right)\right] \times\left[0, Q\left(t^{\beta}\right)\right]$. From (2.6) and (6.8), the marginals of $F\left(C, P\left(t^{\alpha}\right)\right.$, $\left.Q\left(t^{\beta}\right)\right)$ are given by

$$
\begin{aligned}
& F_{U}\left(C, P\left(t^{\alpha}\right), Q\left(t^{\beta}\right)\right)(x)=\frac{C\left(x, Q\left(t^{\beta}\right)\right)}{C\left(P\left(t^{\alpha}\right), Q\left(t^{\beta}\right)\right)}=\frac{t^{\theta} P\left(P^{-1}(x) t^{-\alpha}\right)}{t^{\theta}}=P\left(P^{-1}(x) t^{-\alpha}\right), \\
& F_{V}\left(C, P\left(t^{\alpha}\right), Q\left(t^{\beta}\right)\right)(y)=\frac{C\left(P\left(t^{\alpha}\right), y\right)}{C\left(P\left(t^{\alpha}\right), Q\left(t^{\beta}\right)\right)}=Q\left(t^{-\beta} Q^{-1}(y)\right) .
\end{aligned}
$$

Their inverses are

$$
\begin{aligned}
& F_{U}\left(C, P\left(t^{\alpha}\right), Q\left(t^{\beta}\right)\right)^{-1}(x)=P\left(P^{-1}(x) t^{\alpha}\right), \\
& F_{V}\left(C, P\left(t^{\alpha}\right), Q\left(t^{\beta}\right)\right)^{-1}(y)=Q\left(t^{\beta} Q^{-1}(y)\right) .
\end{aligned}
$$


Assume now that $x$ and $y$ are such that $P^{-1}(x)^{\beta}<Q^{-1}(y)^{\alpha}$. From (6.8) we obtain

$$
F\left(C, P\left(t^{\alpha}\right), Q\left(t^{\beta}\right)\right)(x, y)=\frac{C(x, y)}{C\left(P\left(t^{\alpha}\right), Q\left(t^{\beta}\right)\right)}=\frac{Q^{-1}(y)^{\theta / \beta} P\left(P^{-1}(x) Q^{-1}(y)^{-\alpha / \beta}\right)}{t^{\theta}} .
$$

Thus, for any $(x, y) \in(0,1]^{2}$ such that $P^{-1}\left(F_{U}^{-1}(x)\right)^{\beta} \leq Q^{-1}\left(F_{V}^{-1}(y)\right)^{\alpha}$, i.e. $P^{-1}(x)^{\beta} \leq$ $Q^{-1}(y)^{\alpha}$, we have

$$
\begin{aligned}
\Phi(C, & \left.P\left(t^{\alpha}\right), Q\left(t^{\beta}\right)\right)(x, y) \\
& =F\left(C, P\left(t^{\alpha}\right), Q\left(t^{\beta}\right)\right)\left(F_{U}\left(C, P\left(t^{\alpha}\right), Q\left(t^{\beta}\right)\right)^{-1}(x), F_{V}\left(C, P\left(t^{\alpha}\right), Q\left(t^{\beta}\right)\right)^{-1}(y)\right) \\
& =t^{-\theta}\left(t^{\beta} Q^{-1}(y)\right)^{\theta / \beta} P\left(P^{-1}(x) t^{\alpha}\left(t^{\beta} Q^{-1}(y)\right)^{-\alpha / \beta}\right) \\
& =Q^{-1}(y)^{\theta / \beta} P\left(P^{-1}(x) Q^{-1}(y)^{-\alpha / \beta}\right) \\
& =C(x, y) .
\end{aligned}
$$

Similarly, if $(x, y) \in\left[0, P\left(t^{\alpha}\right)\right] \times\left[0, Q\left(t^{\beta}\right)\right]$ is such that $P^{-1}(x)^{\beta} \geq Q^{-1}(y)^{\alpha}$, then

$$
F\left(C, P\left(t^{\alpha}\right), Q\left(t^{\beta}\right)\right)(x, y)=\frac{P^{-1}(x)^{\theta / \alpha} Q\left(P^{-1}(x)^{-\beta / \alpha} Q^{-1}(y)\right)}{t^{\theta}} .
$$

Thus,

$$
\begin{aligned}
\Phi(C, & \left.P\left(t^{\alpha}\right), Q\left(t^{\beta}\right)\right)(x, y) \\
& =F\left(C, P\left(t^{\alpha}\right), Q\left(t^{\beta}\right)\right)\left(F_{U}\left(C, P\left(t^{\alpha}\right), Q\left(t^{\beta}\right)\right)^{-1}(x), F_{V}\left(C, P\left(t^{\alpha}\right), Q\left(t^{\beta}\right)\right)^{-1}(y)\right) \\
& =t^{-\theta} P^{-1}(x)^{\theta / \alpha} Q\left(P^{-1}(y)^{-\beta / \alpha} Q^{-1}(x)\right) \\
& =C(x, y) .
\end{aligned}
$$

Hence, for all $(x, y) \in[0,1]^{2}$, we have $\Phi\left(C, P\left(t^{\alpha}\right), Q\left(t^{\beta}\right)\right)(x, y)=C(x, y)$, i.e. $C$ is invariant on $\mathcal{D}=\left\{\left(P\left(t^{\alpha}\right), Q\left(t^{\beta}\right)\right): t \in(0,1]\right\}$. This completes the proof of Theorem 4.1.

Proof of Lemma 4.2. (i) Let $C^{*}=\Phi\left(\Phi(C, u, v), u^{*}, v^{*}\right)$. From Lemma 4.1, for $0 \leq x \leq$ $u^{*}$ and $0 \leq y \leq v^{*}$ we have, on the one hand,

$$
\frac{\Phi(C, u, v)(x, y)}{\Phi(C, u, v)\left(u^{*}, v^{*}\right)}=C^{*}\left(\frac{\Phi(C, u, v)\left(x, v^{*}\right)}{\Phi(C, u, v)\left(u^{*}, v^{*}\right)}, \frac{\Phi(C, u, v)\left(u^{*}, y\right)}{\Phi(C, u, v)\left(u^{*}, v^{*}\right)}\right) .
$$

On the other hand, again from Lemma 4.1, we have

$$
\Phi(C, u, v)\left(u^{*}, v^{*}\right)=\Phi(C, u, v)\left(\frac{C\left(u^{\prime}, v\right)}{C(u, v)}, \frac{C\left(u, v^{\prime}\right)}{C(u, v)}\right)=\frac{C\left(u^{\prime}, v^{\prime}\right)}{C(u, v)} .
$$

Furthermore, $F_{U}(C, u, v)^{-1}\left(u^{*}\right)=u^{\prime}$ and $F_{V}(C, u, v)^{-1}\left(v^{*}\right)=v^{\prime}$, by definition of $u^{*}$ and $v^{*}$. Because

$$
\Phi(C, u, v)(x, y)=\frac{C\left(F_{U}(C, u, v)^{-1}(x), F_{V}(C, u, v)^{-1}(y)\right)}{C(u, v)},
$$

it follows, upon dividing (6.11) by (6.10), that

$$
\frac{\Phi(C, u, v)(x, y)}{\Phi(C, u, v)\left(u^{*}, v^{*}\right)}=\frac{C\left(F_{U}(C, u, v)^{-1}(x), F_{V}(C, u, v)^{-1}(y)\right)}{C\left(u^{\prime}, v^{\prime}\right)} .
$$


Let $s=F_{U}(C, u, v)^{-1}(x)$ and $t=F_{V}(C, u, v)^{-1}(y)$. Then substitution into (6.9) yields

$$
\frac{C(s, t)}{C\left(u^{\prime}, v^{\prime}\right)}=C^{*}\left(\frac{C\left(s, v^{\prime}\right)}{C\left(u^{\prime}, v^{\prime}\right)}, \frac{C\left(u^{\prime}, t\right)}{C\left(u^{\prime}, v^{\prime}\right)}\right)
$$

for all $(x, y)$ in $\left[0, u^{*}\right] \times\left[0, v^{*}\right]$. Because $C$ is continuous, $F_{U}(C, u, v)$ and $F_{V}(C, u, v)$ are also continuous, on $[0, u]$ and $[0, v]$ respectively. Hence, $(6.12)$ holds for all $(s, t) \in\left[0, u^{\prime}\right] \times\left[0, v^{\prime}\right]$ because $F_{U}(C, u, v)^{-1}\left(u^{*}\right)=u^{\prime}$ and $F_{V}(C, u, v)^{-1}\left(v^{*}\right)=v^{\prime}$.

Finally, if $0<u^{\prime} \leq u \leq 1$ and $0<v^{\prime} \leq v \leq 1$, then $\Phi\left(C, u^{\prime}, v^{\prime}\right)=\Phi\left(\Phi(C, u, v), u^{*}, v^{*}\right)$, where $u^{*}$ and $v^{*}$ respectively satisfy $u^{*}=C\left(u^{\prime}, v\right) / C(u, v)$ and $v^{*}=C\left(u, v^{\prime}\right) / C(u, v)$.

(ii) Now let $C^{*}=\Phi\left(\Phi(C, u, v), u^{\prime}, v^{\prime}\right)$, which satisfies

$$
\frac{\Phi(C, u, v)(x, y)}{\Phi(C, u, v)\left(u^{\prime}, v^{\prime}\right)}=C^{*}\left(\frac{\Phi(C, u, v)\left(x, v^{\prime}\right)}{\Phi(C, u, v)\left(u^{\prime}, v^{\prime}\right)}, \frac{\Phi(C, u, v)\left(u^{\prime}, y\right)}{\Phi(C, u, v)\left(u^{\prime}, v^{\prime}\right)}\right)
$$

for $0 \leq x \leq u^{\prime}$ and $0 \leq y \leq v^{\prime}$.

Since

$$
\frac{C(x, y)}{C(u, v)}=\Phi(C, u, v)\left(\frac{C(x, v)}{C(u, v)}, \frac{C(u, y)}{C(u, v)}\right),
$$

for all $x \leq u^{\prime}$ and $y \leq v^{\prime}$ we find that

$$
\begin{array}{r}
\frac{C\left(F_{U}(C, u, v)^{-1}(x), F_{V}(C, u, v)^{-1}(y)\right)}{C\left(F_{U}(C, u, v)^{-1}\left(u^{\prime}\right), F_{V}(C, u, v)^{-1}\left(v^{\prime}\right)\right)} \\
=C^{*}\left(\frac{C\left(F_{U}(C, u, v)^{-1}(x), F_{V}(C, u, v)^{-1}\left(v^{\prime}\right)\right)}{C\left(F_{U}(C, u, v)^{-1}\left(u^{\prime}\right), F_{V}(C, u, v)^{-1}\left(v^{\prime}\right)\right)},\right. \\
\left.\frac{C\left(F_{U}(C, u, v)^{-1}\left(u^{\prime}\right), F_{V}(C, u, v)^{-1}(y)\right)}{C\left(F_{U}(C, u, v)^{-1}\left(u^{\prime}\right), F_{V}(C, u, v)^{-1}\left(v^{\prime}\right)\right)}\right) .
\end{array}
$$

Let $u^{*}=F_{U}(C, u, v)^{-1}\left(u^{\prime}\right)$ and $v^{*}=F_{V}(C, u, v)^{-1}\left(v^{\prime}\right)$, which respectively satisfy $C\left(u^{*}, v\right)=u^{\prime} C(u, v)$ and $C\left(u, v^{*}\right)=v^{\prime} C(u, v)$. Then, for all $x \leq u^{*}$ and $y \leq v^{*}$,

$$
\frac{C(x, y)}{C\left(u^{*}, v^{*}\right)}=C^{*}\left(\frac{C\left(x, v^{*}\right)}{C\left(u^{*}, v^{*}\right)}, \frac{C\left(u^{*}, y\right)}{C\left(u^{*}, v^{*}\right)}\right) ;
$$

i.e. $C^{*}=\Phi\left(C, u^{*}, v^{*}\right)$, by Sklar's theorem, since the functions $x \mapsto C\left(x, v^{*}\right) / C\left(u^{*}, v^{*}\right)$ and $y \mapsto C\left(u^{*}, y\right) / C\left(u^{*}, v^{*}\right)$ are continuous.

Finally, if $0<u^{\prime}, u \leq 1$ and $0<v^{\prime}, v \leq 1$, then $\Phi\left(\Phi(C, u, v), u^{\prime}, v^{\prime}\right)=\Phi\left(C, u^{*}, v^{*}\right)$, where $u^{*}$ and $v^{*}$ respectively satisfy $C\left(u^{*}, v\right)=u^{\prime} C(u, v)$ and $C\left(u, v^{*}\right)=v^{\prime} C(u, v)$. Moreover, because $C\left(u^{*}, v\right)=u^{\prime} C(u, v) \leq C(u, v)$ and $x \mapsto C(x, v) / C(u, v)$ is an increasing function, it follows that $u^{*} \leq u$. Similarly $v^{*} \leq v$, which completes the proof of Lemma 4.2.

Proof of Lemma 4.3. To prove the continuity of $\Phi(\cdot, u, v)$, we have to bound differences of the form

$$
\left|\Phi\left(C^{\prime}, u, v\right)(s, t)-\Phi(C, u, v)(s, t)\right|,
$$

where $C, C^{\prime} \in \mathcal{C}$ and $s, t \in[0,1]$. Since the functions $C(\cdot, v) / C(u, v)$ and $C(u, \cdot) / C(u, v)$ are continuous and take the respective values 0 at $u$ and 1 at $v$, we may assume without loss of 
generality that $s=C(x, v) / C(u, v)$ and $t=C(u, y) / C(u, v)$ for some $(x, y) \in[0, u] \times[0, v]$. From Lemma 4.1, it then follows that

$$
\Phi(C, u, v)(s, t)=\frac{C(x, y)}{C(u, v)} .
$$

Now let $\Delta:=C^{\prime}-C$ and consider

$$
\alpha_{C}(x, y):=\frac{C(x, y)}{C(u, v)+\Delta(u, v)} \quad \text { and } \quad \delta_{\Delta}(x, y):=\frac{\Delta(x, y)}{C(u, v)+\Delta(u, v)} .
$$

We obtain

$$
\begin{aligned}
& \frac{C^{\prime}(x, v)}{C^{\prime}(u, v)}=\frac{C(x, v)+\Delta(x, v)}{C(u, v)+\Delta(u, v)}=\alpha_{C}(u, v) s+\delta_{\Delta}(x, v), \\
& \frac{C^{\prime}(u, y)}{C^{\prime}(u, v)}=\alpha_{C}(u, v) t+\delta_{\Delta}(u, y) .
\end{aligned}
$$

Thus, from Lemma 4.1 we obtain

$$
\Phi\left(C^{\prime}, u, v\right)\left(\alpha_{C}(u, v) s+\delta_{\Delta}(x, v), \alpha_{C}(u, v) t+\delta_{\Delta}(u, y)\right)=\frac{C^{\prime}(x, y)}{C^{\prime}(u, v)} .
$$

The expression in (6.13) can be bounded as follows:

$$
\begin{aligned}
\left|\Phi\left(C^{\prime}, u, v\right)(s, t)-\Phi(C, u, v)(s, t)\right| \\
\leq\left|\Phi\left(C^{\prime}, u, v\right)(s, t)-\Phi\left(C^{\prime}, u, v\right)\left(\alpha_{C}(u, v) s+\delta_{\Delta}(x, v), \alpha_{C}(u, v) t+\delta_{\Delta}(u, y)\right)\right| \\
\quad+\left|\Phi\left(C^{\prime}, u, v\right)\left(\alpha_{C}(u, v) s+\delta_{\Delta}(x, v), \alpha_{C}(u, v) t+\delta_{\Delta}(u, y)\right)-\Phi(C, u, v)(s, t)\right| \\
\leq\left|\alpha_{C}(u, v) s+\delta_{\Delta}(x, v)-s\right|+\left|\alpha_{C}(u, v) t+\delta_{\Delta}(u, y)-t\right|+\left|\frac{C^{\prime}(x, y)}{C^{\prime}(u, v)}-\frac{C(x, y)}{C(u, v)}\right| .
\end{aligned}
$$

Here the last inequality follows because any copula is Lipschitz continuous with Lipschitz constant 1, and because of (6.14) and (6.15). Furthermore, from the definitions of $\alpha_{C}$ and $\delta_{\Delta}$ and because $x \leq u$ and $s \leq 1$, we have

$$
\begin{aligned}
\left|\alpha_{C}(u, v) s+\delta_{\Delta}(x, v)-s\right| & =\left|\frac{-\Delta(u, v) s}{C(u, v)+\Delta(u, v)}+\frac{\Delta(x, v)}{C(u, v)+\Delta(u, v)}\right| \\
& \leq \frac{2|\Delta(u, v)|}{C(u, v)+\Delta(u, v)} \\
& \leq \frac{2\|\Delta\|_{\infty}}{C(u, v)-\|\Delta\|_{\infty}},
\end{aligned}
$$

where the denominator on the right-hand side is positive for $C^{\prime}$ sufficiently close to $C$, i.e. if $C^{\prime}(u, v)>\varepsilon>0$ for some $\varepsilon$. Similarly,

$$
\left|\alpha_{C}(u, v) t+\delta_{\Delta}(u, y)-t\right| \leq \frac{2\|\Delta\|_{\infty}}{C(u, v)-\|\Delta\|_{\infty}} .
$$


Furthermore, since $x \leq u$ and $y \leq v$, we have

$$
\begin{aligned}
\left|\frac{C^{\prime}(x, y)}{C^{\prime}(u, v)}-\frac{C(x, y)}{C(u, v)}\right| & =\frac{|\Delta(x, y) C(u, v)-C(x, y) \Delta(u, v)|}{C^{\prime}(u, v) C(u, v)} \\
& \leq \frac{2|C(u, v) \Delta(u, v)|}{C^{\prime}(u, v) C(u, v)} \leq \frac{2\|\Delta\|_{\infty}}{C^{\prime}(u, v)} .
\end{aligned}
$$

From (6.16), (6.17), (6.18), and (6.19), we obtain

$$
\left|\Phi\left(C^{\prime}, u, v\right)(s, t)-\Phi(C, u, v)(s, t)\right| \leq \frac{4\|\Delta\|_{\infty}}{C(u, v)-\|\Delta\|_{\infty}}+\frac{2\|\Delta\|_{\infty}}{C^{\prime}(u, v)},
$$

where the right-hand side is independent of $s$ and $t$ and can be made arbitrarily small as $\|\Delta\|_{\infty}$ becomes small. This completes the proof of Lemma 4.3.

Proof of Theorem 4.2. Let $\left(u_{n}\right)$ and $\left(v_{n}\right)$ be two sequences defined recursively by the following relationship. Let $\alpha$ and $\beta$ be two constants in $(0,1]$, with $(\alpha, \beta) \neq(1,1)$, such that $C\left(u_{n+1}, v_{n}\right) / C\left(u_{n}, v_{n}\right)=\alpha$ and $C\left(u_{n}, v_{n+1}\right) / C\left(u_{n}, v_{n}\right)=\beta$ for all $n \geq 1$, given that $u_{n}$ and $v_{n}$ are strictly positive. Owing to the continuity of $C$, the sequences $\left(u_{n}\right)$ and $\left(v_{n}\right)$ are well defined but not necessarily unique. Those sequences can be defined starting in $(1,1)$, so that $u_{1}=\alpha$ and $v_{1}=\beta$.

Because $\alpha, \beta \in(0,1]$, we have $0 \leq u_{n+1} \leq u_{n}$ and $0 \leq v_{n+1} \leq v_{n}$. Let $u=\lim _{n \rightarrow \infty} u_{n}$ and $v=\lim _{n \rightarrow \infty} v_{n}$. If $u>0$ and $v>0$ then $C(u, v) / C(u, v)=\alpha=\beta$, i.e. $\alpha=\beta=1$, contradicting the hypothesis that $(\alpha, \beta) \neq(1,1)$ and meaning that either $u=0$ or $v=0$.

Consider the copula $C_{n}=\Phi\left(C, u_{n}, v_{n}\right)$. From Lemma 4.2, it follows that

$$
\Phi\left(C, u_{n+1}, v_{n+1}\right)=\Phi\left(\Phi\left(C, u_{n}, v_{n}\right), u_{n+1}^{*}, v_{n+1}^{*}\right),
$$

where $u_{n+1}^{*}=C\left(u_{n+1}, v_{n}\right) / C\left(u_{n}, v_{n}\right)$ and $v_{n+1}^{*}=C\left(u_{n}, v_{n+1}\right) / C\left(u_{n}, v_{n}\right)$. In other words, we have $u_{n+1}^{*}=\alpha$ and $v_{n+1}^{*}=\beta$, whence $\Phi\left(C, u_{n+1}, v_{n+1}\right)=\Phi\left(C_{n}, \alpha, \beta\right)=C_{n+1}$. Thus, $C_{n}=\Phi\left(C, u_{n}, v_{n}\right)$ and as either $u_{n} \rightarrow 0$ or $v_{n} \rightarrow 0$ as $n \rightarrow \infty, C_{n}$ converges towards $C_{0}$ in the same limit. Because, for given $\alpha$ and $\beta, \Phi(\cdot, \alpha, \beta)$ is a continuous function (from Lemma 4.3), $C_{0}$ necessarily satisfies $\Phi\left(C_{0}, \alpha, \beta\right)=C_{0}$. This completes the proof of Theorem 4.2 .

Proof of Corollary 4.1. Since $C_{0}=\lim _{t \rightarrow 0} \Phi(C, r(t), s(t))$, it follows from Theorem 3.1 that $C_{0}(x, y)=\phi\left(\phi_{X}^{-1}(x), \phi_{Y}^{-1}(y)\right)$, where, for $x>0$,

$$
\phi(x, y)=x^{\theta / \alpha} h\left(y x^{-\beta / \alpha}\right), \quad h(x)= \begin{cases}\phi_{Y}(x) & \text { if } x \in[0,1], \\ x^{\theta / \beta} \phi_{X}\left(x^{-\alpha / \beta}\right) & \text { if } x \in(1, \infty) .\end{cases}
$$

In other words,

$$
\begin{aligned}
C_{0}(x, y) & =\phi\left(\phi_{X}^{-1}(x), \phi_{Y}^{-1}(y)\right) \\
& = \begin{cases}\phi_{Y}^{-1}(y)^{\theta / \beta} \phi_{X}\left(\phi_{Y}^{-1}(y)^{-\alpha / \beta} \phi_{X}^{-1}(x)\right) & \text { if } \phi_{X}^{-1}(x)^{\beta}<\phi_{Y}^{-1}(y)^{\alpha}, \\
\phi_{X}^{-1}(x)^{\theta / \alpha} \phi_{Y}\left(\phi_{Y}^{-1}(y) \phi_{X}^{-1}(x)^{-\beta / \alpha}\right) & \text { if } \phi_{X}^{-1}(x)^{\beta} \geq \phi_{Y}^{-1}(y)^{\alpha},\end{cases}
\end{aligned}
$$

i.e. $C_{0}=\Gamma\left(\phi_{X}, \phi_{Y}, \alpha, \beta, \theta\right)$ according to (4.2). This completes the proof of Corollary 4.1. 
Proof of Proposition 4.1. Let $C$ be an absolutely continuous and invariant copula on the unit square. From Lemma 4.1, for all $x, y, u, v \in(0,1]$ we have

$$
\frac{C(x u, y v)}{C(u, v)}=C\left(\frac{C(x u, v)}{C(u, v)}, \frac{C(u, y v)}{C(u, v)}\right) .
$$

Since $C$ is absolutely continuous, differentiating with respect to $x$ and $y$ yields

$$
\frac{u v C_{12}(x u, y v)}{C(u, v)}=\frac{v C_{2}(u, y v)}{C(u, v)} \frac{u C_{1}(x u, v)}{C(u, v)} C_{12}\left(\frac{C(x u, v)}{C(u, v)}, \frac{C(u, y v)}{C(u, v)}\right),
$$

where $C_{1}, C_{2}$, and $C_{12}$ denote the partial derivatives of $C$ with respect to the corresponding variables. This equation can be written as

$$
\frac{C(u, v) C_{12}(x u, y v)}{C_{2}(u, y v) C_{1}(x u, v)}=C_{12}\left(\frac{C(x u, v)}{C(u, v)}, \frac{C(u, y v)}{C(u, v)}\right) .
$$

Inserting $x=y=1$, we obtain

$$
\frac{C(u, v) C_{12}(u, v)}{C_{2}(u, v) C_{1}(u, v)}=C_{12}(1,1)=: \theta+1,
$$

which can be rewritten as

$$
\frac{C_{12}(u, v)}{C_{1}(u, v)}=(\theta+1) \frac{C_{2}(u, v)}{C(u, v)} .
$$

Integrating with respect to $v$ yields

$$
\log C_{1}(u, v)=(\theta+1) \log C(u, v)+\kappa(u),
$$

for some function $\kappa(u)$. In order to determine the function $\kappa$, note that $\log C_{1}(u, 1)=$ $(\theta+1) \log u+\kappa(u)$. Substituting this into (6.20) yields

$$
\log \frac{C_{1}(u, v)}{C_{1}(u, 1)}=(\theta+1) \log \frac{C(u, v)}{u},
$$

and taking the exponential on both sides produces the identity

$$
\begin{aligned}
\frac{-1}{\theta} \frac{\mathrm{d}}{\mathrm{d} u} C(u, v)^{-\theta} & =\frac{C_{1}(u, v)}{C(u, v)^{\theta+1}}=\frac{C_{1}(u, 1)}{u^{\theta+1}}=\frac{C_{1}(u, 1)}{C(u, 1)^{\theta+1}}=\frac{-1}{\theta} \frac{\mathrm{d}}{\mathrm{d} u} C(u, 1)^{-\theta} \\
& =\frac{-1}{\theta} \frac{\mathrm{d}}{\mathrm{d} u} u^{-\theta}
\end{aligned}
$$

Integrating with respect to $u$ yields

$$
C(u, v)^{-\theta}=u^{-\theta}+\lambda(v)
$$

for some function $\lambda(v)$. Setting $u=1$, we see that $\lambda(v)=v^{-\theta}-1$, whence $C(u, v)=$ $\left(u^{-\theta}+v^{-\theta}-1\right)^{-1 / \theta}$, which is the Clayton copula with parameter $\theta$. Conversely, since the Clayton copula is absolutely continuous and also invariant on $[0,1]^{2}$, it follows that it is the only copula with these properties. 


\section{References}

ACZÉL, J. (1966). Lectures on Functional Equations and Their Applications. Academic Press, New York.

BäUerle, N. AND Müller, A. (1998). Modeling and comparing dependencies in multivariate risk portfolios. ASTIN Bull. 28, 59-76.

Bingham, N. H., Goldie, C. M. and Teugels, J. L. (1987). Regular Variation. Cambridge University Press.

Brémaud, P. (1981). Point Processes and Queues. Springer, New York.

Clayton, D. G. (1978). A model for association in bivariate life tables and its application in epidemiological studies of familial tendency in chronic disease incidence. Biometrika $\mathbf{6 5}, 141-151$.

Coles, S. G. And Tawn, J. A. (1991). Modelling extreme multivariate events. J. R. Statist. Soc. B 53, 377-392.

Coles, S. G. And TAwn, J. A. (1994). Statistical methods for multivariate extremes: an application to structural design. J. R. Statist. Soc. C 43, 1-48 (with discussion).

Cook, R. D. AND Johnson, M. E. (1981). A family of distributions for modelling non-elliptically symmetric multivariate data. J. R. Statist. Soc. B 43, 210-218.

Daul, S., De Giorgi, E., Lindskog, F. and McNeil, A. (2003). Using the grouped $t$-copula. Risk 16, 73-76.

DAvis, M. AND Lo, V. (2001a). Infectious defaults. Quantitative Finance 1, 382-387.

Davis, M. AND Lo, V. (2001b). Modelling default correlation in bond portfolios. In Mastering Risk. Volume 2 Applications, ed. C. Alexander, Financial Times/Prentice Hall, Harlow, pp. 141-151.

De HaAn, L. and Resnick, S. I. (1977). Limit theory for multivariate sample extremes. Z. Wahrscheinlichkeitsth. 40, 317-337.

De HaAn, L., Omey, E. and Resnick, S. I. (1984). Domains of attraction of regular variation in $\boldsymbol{R}^{d}$. J. Multivariate Anal. 14, 17-33.

Dhaene, J. and Denuit, M. (1999). The safest dependence structure among risks. Insurance Math. Econom. 25, $11-21$.

Dhaene, J. And Goovaerts, M. J. (1996). Dependency of risks and stop-loss order. ASTIN Bull. 26, 201-212.

Embrechts, P., KlüPPelberg, C. ANd Mikosch, T. (1997). Modelling Extremal Events for Insurance and Finance. Springer, Berlin.

Embrechts, P., McNeil, A. J. And Straumann, D. (2002). Correlation and dependence in risk management: properties and pitfalls. In Risk Management: Value at Risk and Beyond, ed. M. A. H. Dempster, Cambridge University Press, pp. 176-223.

Fredricks, G. A., Nelsen, R. B. And Rodriguez-Lallena, J. A. (2005). Copulas with fractal supports. Insurance Math. Econom. 37, 42-48.

Frees, E. W. And Valdez, E. A. (1998). Understanding relationships using copulas. N. Amer. Actuar. J. 2, 1-25.

JoE, H. (1997). Multivariate Models and Dependence Concepts. Chapman and Hall, London.

JuRI, A. AND WÜThrich, M. V. (2002). Copula convergence theorems for tail events. Insurance Math. Econom. 30, 405-420.

JURI, A. AND WÜThrich, M. V. (2004). Tail dependence from a distributional point of view. Extremes 6, $213-246$.

Lando, D. (1998). On Cox processes and credit risky securities. Rev. Derivatives Res. 2, 99-120.

Nelsen, R. B. (1999). An Introduction to Copulas. Springer, New York.

Resnick, S. (1987). Extreme Values, Regular Variation, and Point Processes. Springer, New York.

Schönbucher, P. And Schubert, D. (2001). Copula-dependent default risk in intensity models. Preprint. Available at http://www.math.ethz.ch/ schonbuc/.

SkLar, A. (1959). Fonctions de répartition à $n$ dimensions et leurs marges. Publ. Inst. Statist. Univ. Paris 8, $229-231$. 